\title{
Advancing LHC probes of dark matter from the inert two-Higgs-doublet model with the monojet signal
}

\author{
A. Belyaev and S. Moretti \\ Particle Physics Department, Rutherford Appleton Laboratory, \\ Chilton, Didcot, Oxon OX11 0QX, United Kingdom \\ and School of Physics and Astronomy, University of Southampton, \\ Southampton SO17 1BJ, United Kingdom \\ T. R. Fernandez Perez Tomei and S. F. Novaes \\ Universidade Estadual Paulista, R. Dr. Bento Teobaldo Ferraz, 271-Várzea da Barra Funda, \\ São Paulo, São Paulo 01140-070 Brazil \\ P. G. Mercadante \\ Universidade Federal do ABC, Avenida dos Estados, 5001, Bangú \\ Santo Andre, Sao Paulo 09210-580. Brazil \\ C. S. Moon \\ Kyungpook National University, Daegu 41566, Korea \\ L. Panizzi \\ Department of Physics and Astronomy, Uppsala University, Box 516, SE-751 20 Uppsala, Sweden \\ and School of Physics and Astronomy, University of Southampton, Southampton SO17 1BJ, United Kingdom \\ F. Rojas \\ Universidad Técnica Federico Santa María, Avenida España 1680, Valparaíso, Chile \\ and School of Physics and Astronomy, University of Southampton, Southampton SO17 1BJ, United Kingdom \\ M. Thomas \\ School of Physics and Astronomy, University of Southampton, Southampton SO17 1BJ, United Kingdom
}

(Received 27 September 2018; published 7 January 2019)

The inert two-Higgs-doublet Model (i2HDM) is a well-motivated minimal consistent dark matter (DM) model, but it is rather challenging to test at the LHC in the parameter space allowed by relic density and DM direct detection constraints. This is especially true when considering the latest XENON $1 \mathrm{~T}$ data on direct DM searches, which we use here to present the best current combined limit on the i2HDM parameter space. In this analysis, we present prospects to advance the exploitation of DM monojet signatures from the $\mathrm{i} 2 \mathrm{HDM}$ at the $\mathrm{LHC}$, by emphasising that a shape analysis of the missing transverse momentum distribution allows one to sizably improve the LHC discovery potential. For a key element of our analysis, we explore the validity of using an effective vertex, $g g H$, for the coupling of the Higgs boson to gluons using a full one-loop computation. We have found sizeable differences between the two approaches, especially in the high missing transverse momentum region, and incorporated the respective K-factors to obtain the correct kinematical distributions. As a result, we delineate a realistic search strategy and present the improved current and projected LHC sensitivity to the i2HDM parameter space.

DOI: $10.1103 /$ PhysRevD.99.015011

Published by the American Physical Society under the terms of the Creative Commons Attribution 4.0 International license. Further distribution of this work must maintain attribution to the author(s) and the published article's title, journal citation, and DOI. Funded by SCOAP.

\section{INTRODUCTION}

Despite several independent evidences of dark matter $(\mathrm{DM})$ at the cosmological scale, its nature remains unknown since no experiment so far has been able to claim its detection in the laboratory and probe its 
properties. Potentially, DM can be probed in direct or indirect detection experiments as well as be produced at the LHC or future machines, though the latter can only detect DM candidates, as any observed missing energy can still be interpreted as generated by long-lived neutral particles. This combined effort on advancing our knowledge of DM properties is one of the key goals of the astroparticle and high energy physics communities.

A convenient way to understand the potential of both collider and noncollider experiments to probe DM is to explore simple, fully calculable, renormalizable models with viable DM candidates, which we refer to as minimal consistent dark matter (MCDM) models. We do not know yet which theoretical scenario corresponds to reality, but any model of this kind offers an excellent opportunity to gain insight into the intricate interplay between collider and noncollider constraints. MCDM models, which can be viewed as robust toy models, are self-consistent and can be easily incorporated into larger theoretically driven scenarios of physics beyond the Standard Model. Because of their attractive features, MCDM models can be considered as the next step beyond DM effective field theory (EFT) (see e.g. Refs. [1-13]) and simplified DM models (see e.g. Refs. [14-21]).

The inert two-Higgs-doublet model (i2HDM), which was initially suggested more than 30 years ago in Ref. [22], is one of the most representative MCDM models which has become very attractive lately [23-45] in the light of intensive DM searches. In fact, besides providing a good DM candidate, the i2HDM can also give rise to an "improved naturalness" [24] since large radiative corrections from the inert Higgs sector can screen the SM Higgs contribution to the eletroweak (EW) parameter $\Delta T$.

It was shown in Ref. [45] that the LHC has limited sensitivity to probe the i2HDM with the monojet signature using the cut-based analyses optimized for the lowluminosity Run 2 data. To complement these studies, in the present paper, we explore the LHC potential to probe $\mathrm{DM}$ via the monojet signature in the $\mathrm{i} 2 \mathrm{HDM}$ scenario by exploiting a larger amount of information from observables at the differential level. More specifically, we will consider the shape of the missing transverse momentum $\left(E_{\mathrm{T}}^{\text {miss }}\right)$ distribution. New findings of this study include a) updating limits on the i2HDM parameter space following the recent XENON 1T results on DM direct detection (DD) searches; b) exploring the range of validity of the effective $g g H$ vertex in the heavy top mass limit by considering the $E_{\mathrm{T}}^{\text {miss }}$ distribution and comparing its shape to the full one-loop result, which will allow us to determine a realistic LHC potential for probing DM in different kinematical regions; c) optimizing and improving the LHC sensitivity to the DM monojet signal from the i2HDM defined by Higgs and $Z$-boson mediation processes using a shape analysis of the $E_{\mathrm{T}}^{\text {miss }}$ distribution; d) projecting our results to the High Luminosity LHC (HL-LHC) phase.
The rest of the paper is organized as follows. In Sec. II, we discuss the i2HDM parameter space together with the current status of theoretical and experimental constraints. In Sec. III, we present the main results of the paper, which include the analysis of the validity of the effective $g g H(H$ being the SM-like Higgs) vertex approach, the exploration of several model benchmarks, and finally finding the LHC potential to probe the i2HDM at present and projected luminosities via exploitation of the $E_{\mathrm{T}}^{\text {miss }}$ shape in the monojet signature. In Sec. IV, we draw our conclusions.

\section{I2HDM}

\section{A. Parameter space}

The i2HDM [22-25] is an extension of the Standard Model (SM) with a second scalar doublet $\phi_{2}$ possessing the same quantum numbers as the SM Higgs doublet $\phi_{1}$ but with no couplings to fermions, thus providing its inert nature. This construction is protected by a discrete $\mathcal{Z}_{2}$ symmetry under which $\phi_{2}$ is odd and all the other fields are even. The Lagrangian of the scalar sector is

$$
\mathcal{L}=\left|D_{\mu} \phi_{1}\right|^{2}+\left|D_{\mu} \phi_{2}\right|^{2}-V\left(\phi_{1}, \phi_{2}\right)
$$

where $V$ is the potential with all scalar interactions compatible with the $\mathcal{Z}_{2}$ symmetry:

$$
\begin{aligned}
V= & -m_{1}^{2}\left(\phi_{1}^{\dagger} \phi_{1}\right)-m_{2}^{2}\left(\phi_{2}^{\dagger} \phi_{2}\right)+\lambda_{1}\left(\phi_{1}^{\dagger} \phi_{1}\right)^{2}+\lambda_{2}\left(\phi_{2}^{\dagger} \phi_{2}\right)^{2} \\
& +\lambda_{3}\left(\phi_{1}^{\dagger} \phi_{1}\right)\left(\phi_{2}^{\dagger} \phi_{2}\right)+\lambda_{4}\left(\phi_{2}^{\dagger} \phi_{1}\right)\left(\phi_{1}^{\dagger} \phi_{2}\right) \\
& +\frac{\lambda_{5}}{2}\left[\left(\phi_{1}^{\dagger} \phi_{2}\right)^{2}+\left(\phi_{2}^{\dagger} \phi_{1}\right)^{2}\right] .
\end{aligned}
$$

In the unitary gauge, the doublets take the form

$$
\phi_{1}=\frac{1}{\sqrt{2}}\left(\begin{array}{c}
0 \\
v+H
\end{array}\right), \quad \phi_{2}=\frac{1}{\sqrt{2}}\left(\begin{array}{c}
\sqrt{2} h^{+} \\
h_{1}+i h_{2}
\end{array}\right),
$$

where we consider the parameter space in which only the first, SM-like doublet acquires a vacuum expectation value, $v$. In the notation $\left\langle\phi_{i}^{0}\right\rangle=v_{i} / \sqrt{2}$, this inert minimum corresponds to $v_{1}=v, v_{2}=0$. After EW symmetry breaking, the $\mathcal{Z}_{2}$ symmetry is still conserved by the vacuum state, which forbids direct coupling of any single inert field to the SM fields and protects the lightest inert boson from decaying, hence providing the DM candidate in this scenario. In contrast, the interactions of a pair of inert scalars with the SM gauge bosons and SM-like Higgs $H$ are allowed, thus giving rise to various signatures at colliders and at DM detection experiments.

In addition to the SM-like scalar $H$, the model contains one inert charged $h^{ \pm}$and two further inert neutral $h_{1}, h_{2}$ scalars. The two neutral scalars of the i2HDM have 
opposite $C P$ parities, but it is impossible to unambiguously determine which of them is $C P$ even and which one is $C P$ odd since the model has two $C P$ symmetries, $h_{1} \rightarrow h_{1}$, $h_{2} \rightarrow-h_{2}$ and $h_{1} \rightarrow-h_{1}, h_{2} \rightarrow h_{2}$, which get interchanged upon a change of basis $\phi_{2} \rightarrow i \phi_{2}$. This makes the specification of the $C P$ properties of $h_{1}$ and $h_{2}$ a basis-dependent statement. Therefore, following Ref. [45], we denote the two neutral inert scalar masses as $M_{h_{1}}<M_{h_{2}}$, without specifying which is scalar or pseudoscalar, so that $h_{1}$ is the DM candidate.

The model can be conveniently described by a fivedimensional parameter space [45] using the following phenomenologically relevant variables,

$M_{h_{1}}, \quad M_{h_{2}}>M_{h_{1}}, \quad M_{h^{+}}>M_{h_{1}}, \quad \lambda_{2}>0$,

$\lambda_{345}>-2 \sqrt{\lambda_{1} \lambda_{2}}$,

where $M_{h_{1}}, M_{h_{2}}$, and $M_{h^{+}}$are the masses of the two neutral and charged inert scalars, respectively, whereas $\lambda_{345}=\lambda_{3}+\lambda_{4}+\lambda_{5}$ is the coupling which governs the Higgs-DM interaction vertex $H h_{1} h_{1}$. The masses of the physical scalars are expressed in terms of the parameters of the Lagrangian in Eqs. (1) and (2) as follows:

$$
\begin{aligned}
M_{\mathrm{H}}^{2} & =2 \lambda_{1} v^{2}=2 m_{1}^{2}, \\
M_{h^{+}}^{2} & =\frac{1}{2} \lambda_{3} v^{2}-m_{2}^{2}, \\
M_{h_{1}}^{2} & =\frac{1}{2}\left(\lambda_{3}+\lambda_{4}-\left|\lambda_{5}\right|\right) v^{2}-m_{2}^{2}, \\
M_{h_{2}}^{2} & =\frac{1}{2}\left(\lambda_{3}+\lambda_{4}+\left|\lambda_{5}\right|\right) v^{2}-m_{2}^{2}>M_{h_{1}}^{2} .
\end{aligned}
$$

\section{B. Theoretical and experimental constraints}

Constraints on the Higgs potential from requiring vacuum stability and a global minimum take the following form [45],

$$
\begin{cases}M_{h_{1}}^{2}>0(\text { the trivial one }) & \text { for }|R|<1, \\ M_{h_{1}}^{2}>\left(\lambda_{345} / 2 \sqrt{\lambda_{1} \lambda_{2}}-1\right) \sqrt{\lambda_{1} \lambda_{2}} v^{2}=(R-1) \sqrt{\lambda_{1} \lambda_{2}} v^{2} & \text { for } R>1\end{cases}
$$

where $R=\lambda_{345} / 2 \sqrt{\lambda_{1} \lambda_{2}}$ and $\lambda_{1} \approx 0.129$ is fixed as in the SM by the Higgs mass in Eq. (5). The latter condition places an important upper bound on $\lambda_{345}$ for a given DM mass $M_{h_{1}}$.

The theoretical upper limit on $\lambda_{345}$ for a given DM mass comes from the vacuum stability constraint. Using Eq. (17) from Ref. [45] and an upper limit on $\lambda_{2}$ (which is about $4 \pi / 3$ for DM masses below $300 \mathrm{GeV}$ ), we find

$$
\lambda_{345}<2\left(\frac{M_{h_{1}}^{2}}{v^{2}}+\sqrt{\lambda_{1} \lambda_{2}^{\max }}\right) \simeq 2\left(\frac{M_{h_{1}}^{2}}{v^{2}}+\sqrt{\lambda_{1} \frac{4 \pi}{3}}\right) .
$$

When $M_{h_{1}}<M_{\mathrm{H}} / 2, \lambda_{345}$ has a much stronger limit coming from the invisible Higgs boson decay measured at the LHC. In this region, the limit on $\left|\lambda_{345}\right|$ can be written in the following form,

$$
\left|\lambda_{345}\right|<\left(\frac{8 \pi g_{W}^{2} \Gamma_{\mathrm{SM}} M_{\mathrm{H}}}{M_{W}^{2}\left(\frac{1}{\mathrm{Br}(H \rightarrow \text { invis })}-1\right) \sqrt{1-4 \frac{M_{h_{1}}^{2}}{M_{\mathrm{H}}^{2}}}}\right)^{1 / 2},
$$

where $\operatorname{Br}(H \rightarrow$ invis $)$ is the experimental limit on the branching ratio $(\mathrm{Br})$ for invisible Higgs boson decays, $\Gamma_{\mathrm{SM}}$ is the SM-like Higgs boson width, and $g_{W}$ is the SM weak coupling. This formula is derived under the assumption that $H \rightarrow h_{1} h_{1}$ is the only invisible channel of the SM-like Higgs boson. In Fig. 1, we present values of $\left|\lambda_{345}\right|_{\max }$ as a function of $M_{h_{1}}$ for several values of $\operatorname{Br}(H \rightarrow$ invis) including 0.25 and 0.24 corresponding to the most up-to-date limits on $\operatorname{Br}(H \rightarrow$ invis $)$ from ATLAS [46] and CMS [47], respectively. One should note that experimental limits on $\operatorname{Br}(H \rightarrow$ invis $)$ are actually placed for $H \rightarrow$ invis to any channel and thus also include $H \rightarrow Z Z \rightarrow$ neutrinos, which is, however, below the per mille level and can thus be neglected in our study.

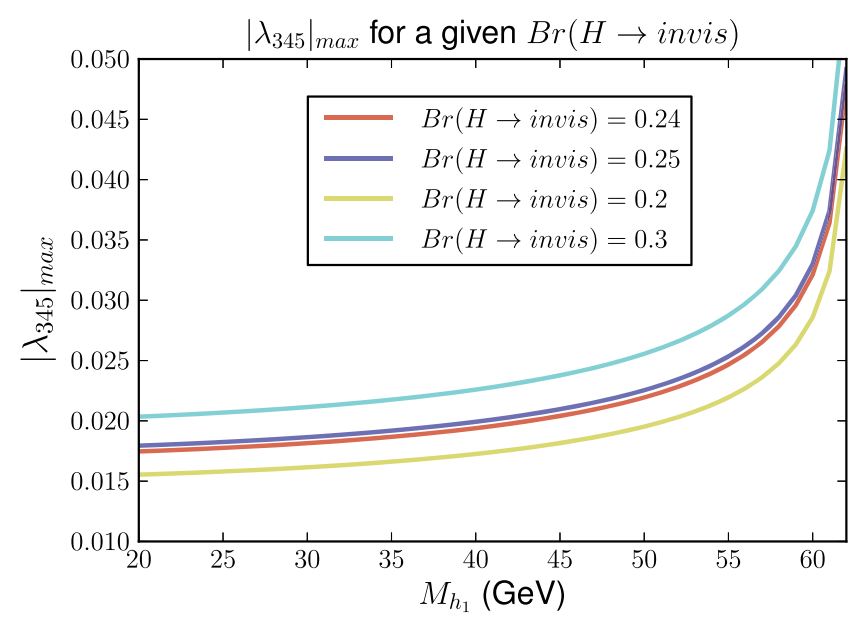

FIG. 1. The values of $\left|\lambda_{345}\right|_{\max }$ as a function of $M_{h_{1}}$ for selected choices of $\operatorname{Br}(H \rightarrow$ invis $)$. This limit is found under the assumption that $H \rightarrow h_{1} h_{1}$ is the only invisible decay channel of the SM-like Higgs boson. 

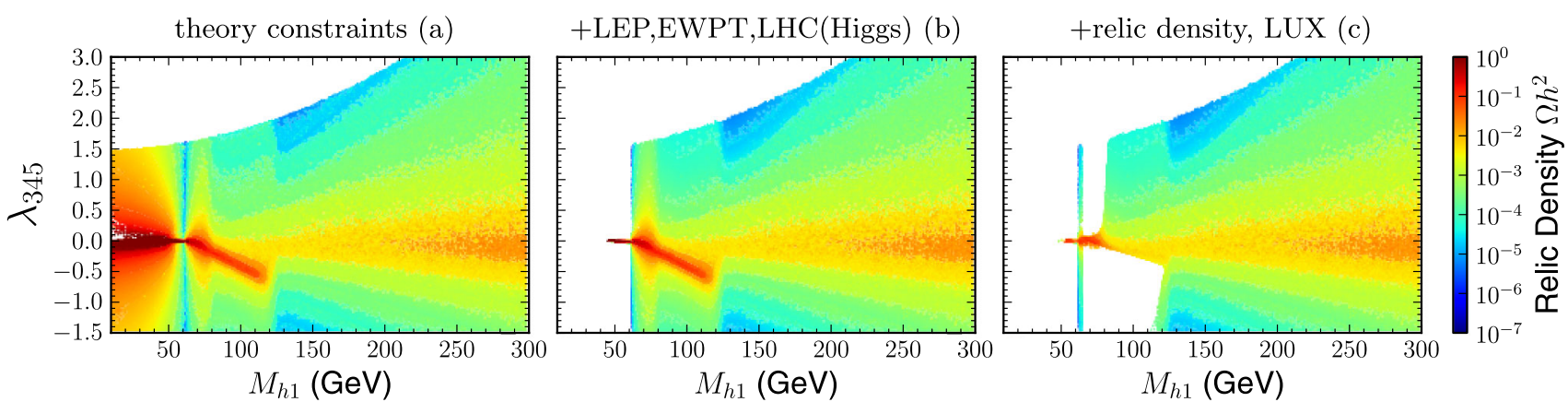

FIG. 2. Color maps of DM relic abundance projected on the plane $\left(M_{h_{1}}, \lambda_{345}\right)$ from Ref. [45]. The three plots correspond to the surviving points after the sequential application of the sets of constraints described in the text.

The $\left|\lambda_{345}\right|_{\max }$ value increases when $M_{h_{1}}$ approaches $M_{\mathrm{H}} / 2$, ranging from 0.024 at $M_{h_{1}}=50 \mathrm{GeV}$ to 0.053 at $M_{h_{1}}=62 \mathrm{GeV}$. At the same time, the $\Omega h^{2}<0.1$ constraint sets the lower limit $M_{h_{1}} \gtrsim 40 \mathrm{GeV}$ since below it there are no effective annihilation and/or co-annihilation DM channels to bring DM relic density to a low enough level consistent with Planck constraints. One should note that when the decay $H \rightarrow h_{2} h_{2}$ also takes place and when $h_{1}$ and $h_{2}$ are close in mass (below, say a few giga-electronvolts) this channel will also contribute to the invisible Higgs decay. In this case, the limit on $\lambda_{345}$ can be easily modified, taking into account that $\lambda_{H h_{2} h_{2}}=\lambda_{345}+\frac{M_{h_{2}}^{2}-M_{h_{1}}^{2}}{v^{2}}$, and thus for $M_{h_{2}} \simeq M_{h_{1}}$, one has $\lambda_{H h_{2} h_{2}} \simeq \lambda_{H h_{1} h_{1}}=\lambda_{345}$.

The comprehensive analysis of the i2HDM parameter space performed in Ref. [45] using an i2HDM implementation into the CALCHEP [48] and MICROMEGAs [49,50] frameworks demonstrates an important complementarity of various constraints, which is presented in Fig. 2 as an effect of the sequential application of a) theoretical constraints from vacuum stability, perturbativity, and unitarity (theory); b) experimental constraints from colliders [Large ElectronPositron collider (LEP) and LHC Higgs data, including those from EW precision test (EWPT) data]; and c) the upper bound on the DM relic density at $\Omega_{\mathrm{DM}} h^{2}$ given by Planck [51,52] and constraints from DM DD searches at LUX [53].

From Figs. 2(a) and 2(b), one can see the large effect of the invisible Higgs decay constraint on $\lambda_{345}$ (of the order of $10^{-2}$ ) in the $M_{h_{1}}<M_{\mathrm{H}} / 2$ region, which is 2 orders of magnitude stronger than the constraint on $\lambda_{345}$ from vacuum stability. The constraint from DM DD searches from LUX [53] further limits $\lambda_{345}$ as one can see from Fig. 2(c). Let us recall first that we use the rescaled DD spin-independent (SI) cross section, $\hat{\sigma}_{\mathrm{SI}}=R_{\Omega} \times \sigma_{\mathrm{SI}}$, where the scaling factor $R_{\Omega}=\Omega_{\mathrm{DM}} / \Omega_{\mathrm{DM}}^{\text {Planck }}$ takes into account the case of $h_{1}$ representing only a part of the total DM budget, thus allowing for a convenient comparison of the model predictions with the DM DD limits. One can see that this constraint is not symmetric with respect to the sign of $\lambda_{345}$; the parameter space with $\lambda_{345}<0$ receives stronger

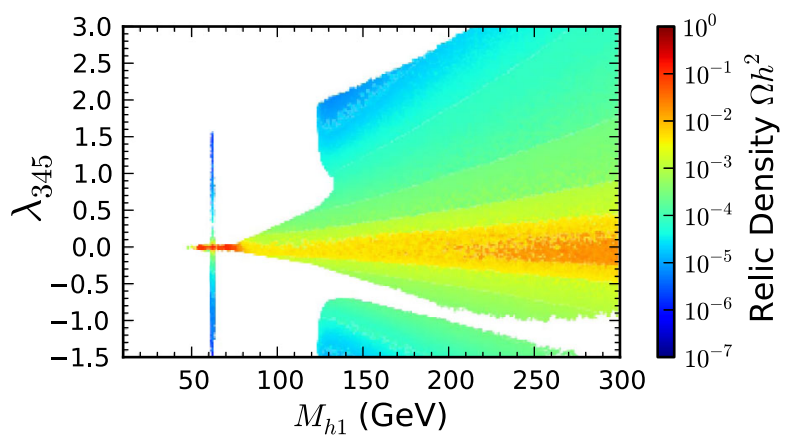

FIG. 3. The new constraints on the i2HDM parameter space from XENON1T searches for DM [54].

constraints. The reason for this is that the sign of $\lambda_{345}$ defines the sign of the interference of DM annihilation into EW gauge bosons via the Higgs boson and via the $h_{1} h_{1} V V$ quartic coupling. For positive $\lambda_{345}$, the interference is positive, and the relic density is correspondingly lower, so the DM DD rates rescaled with relic density, $\hat{\sigma}_{\mathrm{SI}}$, are lower than for the case of negative $\lambda_{345}$, when the corresponding interference is negative and the relic density is higher. One should also note that the combined constraints exclude $M_{h_{1}}<45 \mathrm{GeV}$ for the whole parameter space of the i2HDM.

Since DM DD constraints play an important role, in the light of recent results from the XENON1T experiment [54], we have performed a further comprehensive scan of the i2HDM parameter space analogously to Ref. [45] and have found new constraints. ${ }^{1}$ Our results are shown in Fig. 3, where we present the i2HDM parameter space left after the application of theory, LEP, EWPT, LHC constraints as well as upper bounds on the relic density from Planck and DM DD limits from XENON1T. One can see a large effect of the XENON1T constraints on $\lambda_{345}$, which improve LUX limits by more than 1 order of magnitude, chiefly, over the $M_{\mathrm{H}} / 2<M_{h_{1}}<125 \mathrm{GeV}$ region. In particular, in this region, $\left|\lambda_{345}\right|$ is limited to be always

${ }^{1}$ For the XENON1T limit, we have used digitized data from the PhenoData database [55]. 

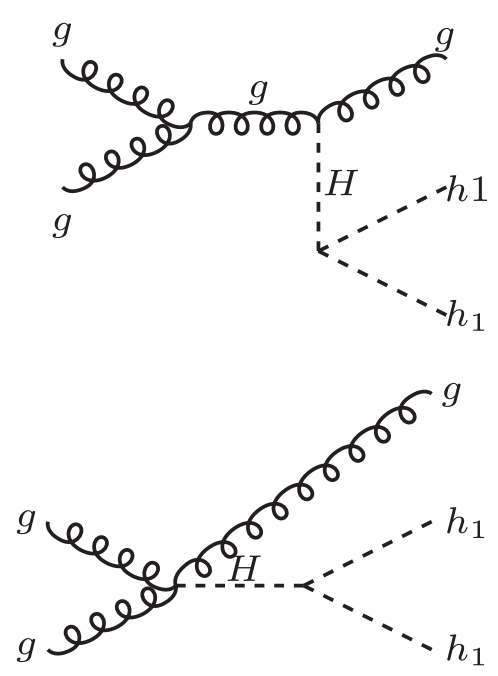
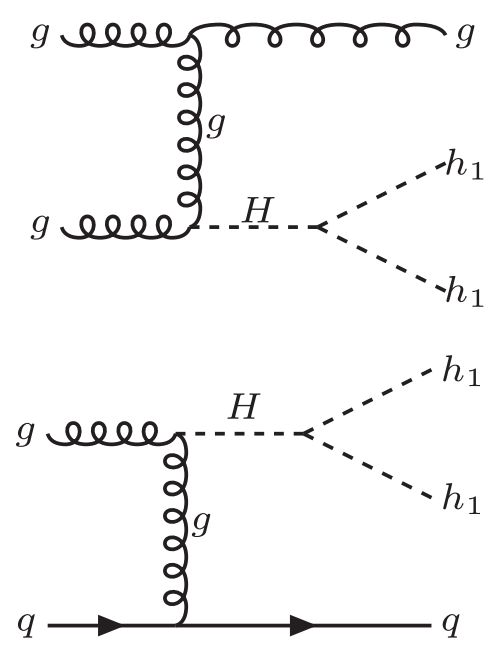
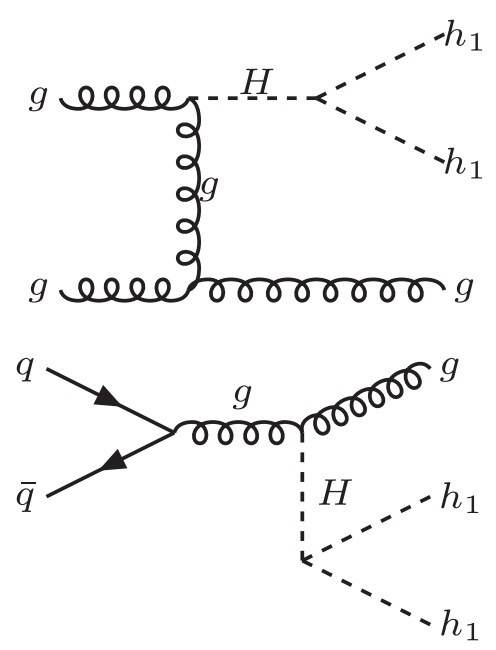

FIG. 4. Feynman diagrams for the $g g \rightarrow h_{1} h_{1}+g$ process contributing to the monojet signature.

below about 0.05 , which is crucial for one of the main signatures of DM searches at the LHC which we discuss below. $^{2}$

The asymmetric picture with respect to negative and positive values of $\lambda_{345}$ is even more pronounced in the case of these latest results as one can clearly see the white funnel region excluded for $\lambda_{345}<0$. The reason for this is again the negative interference between DM annihilation into EW gauge bosons via Higgs boson exchange and $h_{1} h_{1} V V$ quartic couplings described above; in this funnel region, this negative interference brings the DM relic density up, which in turn increases the DM DD rates.

One should note that, though constraints from DM DD and invisible Higgs decay on $\left|\lambda_{345}\right|$ dominate the one from vacuum stability, the latter sets the most strict upper bound on $\lambda_{345}$ for $M_{h_{1}} \simeq M_{\mathrm{H}} / 2$. In this region, the invisible Higgs decay is suppressed by the phase space, while DM DD rates rescaled by relic density are suppressed because $\Omega h^{2}$ is driven to low values in this parameter space, which is dominated by $h_{1} h_{1} \rightarrow H$ resonant annihilation. Therefore, the constraint from vacuum stability which becomes important in this region limits $\lambda_{345} \lesssim 1.6$ as follows from Eq. (7).

\section{MONOJET SIGNATURES AT THE LHC}

The i2HDM exhibits different collider signatures, which can potentially be accessible at the LHC. In this analysis,

\footnotetext{
${ }^{2}$ One should note that in Ref. [56] the authors have also analysed i2HDM parameter space using XENON1T (2017) constraints. However, the pattern of their surviving parameter space is quite different in some specific regions. For example, for $M_{h 1}$ just above $M_{\mathrm{H}} / 2$, we have found parameter space with $\lambda_{345} \simeq 1, M_{h 1} \simeq M_{h 2}$, which satisfy experimental limits and which have DM relic density below the Planck upper limit primarily because of the strong $h_{1}-h_{2}$ coannihilation channel. We believe that this region was missed in Ref. [56].
}

we will focus on monojet final states, which arise from $g g \rightarrow h_{1} h_{1}+g, q g \rightarrow h_{1} h_{1}+q$, and $q \bar{q} \rightarrow h_{1} h_{1}+g$ processes, to which we will refer cumulatively as the $h_{1} h_{1} j$ process. The corresponding Feynman diagrams are presented in Fig. 4.

For this signature, and for $M_{h_{1}}>M_{\mathrm{H}} / 2$, the relevant nontrivial parameter space is one dimensional and corresponds to the DM mass, $M_{h_{1}}$, since the production cross section is proportional to $\left(\lambda_{345}\right)^{2}$. For $M_{h_{1}}<M_{\mathrm{H}} / 2$, however, the situation can be different, for two reasons: a) only $H \rightarrow h_{1} h_{1}$ takes place, so that the cross section is defined by the production of the SM-like Higgs times $\operatorname{Br}\left(H \rightarrow h_{1} h_{1}\right)$, which is a function of $\lambda_{345}$ and $M_{h_{1}}$, and b)

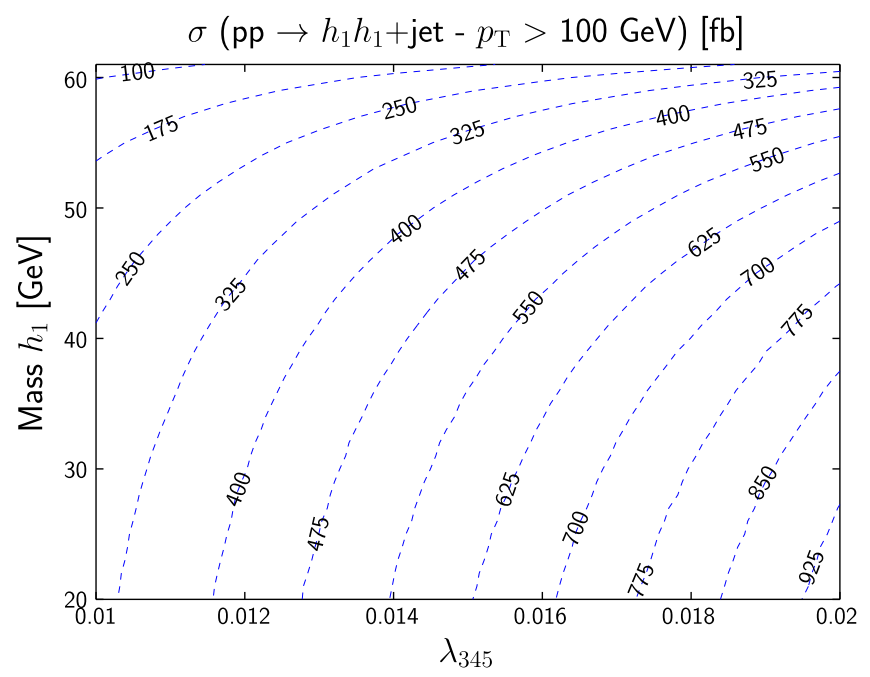

FIG. 5. Cross sections vs DM mass $M_{h_{1}}$ and coupling constant $\lambda_{345}$ for the monojet process $h_{1} h_{1} j$ at the LHC@13 TeV. The mass of the $h_{2}$ particle is set to $M_{h_{2}}=200 \mathrm{GeV}$. Here, the cross section was evaluated for the initial cut on $p_{\mathrm{T}}^{\text {jet }}>100 \mathrm{GeV}$. 

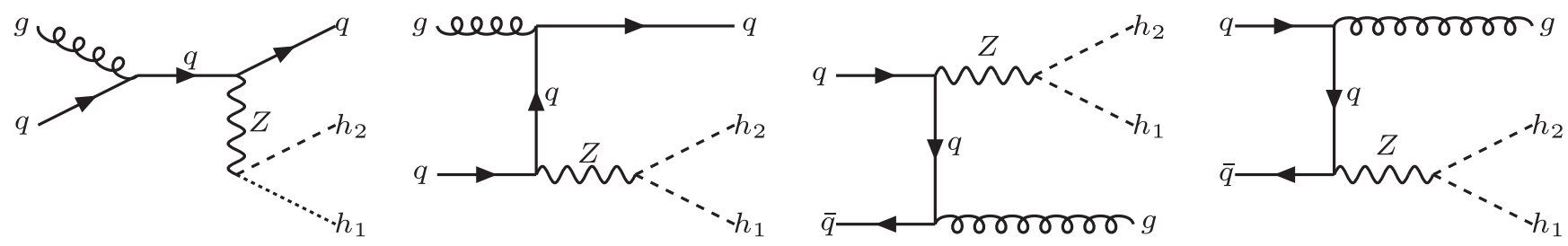

FIG. 6. Feynman diagrams for the $q \bar{q} \rightarrow h_{1} h_{2}+g\left(g q \rightarrow h_{1} h_{2}+q\right)$ process.

both $H \rightarrow h_{1} h_{1}$ and $H \rightarrow h_{2} h_{2}$ contribute to the invisible Higgs decay, which then implies that both $h_{1} h_{1} j$ and $h_{2} h_{2} j$ will contribute to the same signature (for a few gigaelectron-volts mass difference between $h_{2}$ and $h_{1}, h_{2} \rightarrow$ $h_{1} f \bar{f}$ is invisible because of the soft fermions $f$ in the final state), the cross section of which is defined by the production of the SM-like Higgs state times $\left(\operatorname{Br}\left(H \rightarrow h_{1} h_{1}\right)+\operatorname{Br}\left(H \rightarrow h_{2} h_{2}\right)\right.$, which is a function of $\lambda_{345}, M_{h_{1}}$ as well as $M_{h_{2}}$.

In Fig. 5, we present the cross sections for the monojet process $h_{1} h_{1} j$ at the LHC@ $13 \mathrm{TeV}$ in the $\left(M_{h_{1}}, \lambda_{345}\right)$ plane. The monojet cross section was evaluated with the initial cut on $p_{\mathrm{T}}^{\text {jet }}>100 \mathrm{GeV}, \lambda_{345}$ has been chosen to be in the range $[0.01,0.02], M_{h_{1}}$ has been chosen in the range [20, 60] $\mathrm{GeV}$, and $M_{h_{2}}$ has been fixed to $200 \mathrm{GeV}$. We can see that, for this range of parameters, the cross section rate is between 100 and $1000 \mathrm{fb}$, which gives us a strong motivation to probe this signal at the LHC. For this and the following parton level calculations and simulations, we have used the HEPMDB site [57], the CALCHEP package [48], and the NNPDF23LO (AS_0130_QED) parton distribution function (PDF) set [58] with both the factorization and renormalization scales set to the transverse mass of the final state particles.

An important remark is that the mass of the top quark in the loop which defines the $g g H$ coupling can be less than the energy scale of the $h_{1} h_{1} j$ process which is related to the jet transverse momentum, $p_{T}^{\text {jet }}$. Hence, in the region of high $p_{T}^{\text {jet }}$, one should check the validity of the EFT approach based on the heavy top-quark approximation, which is often used for simplification. This is the subject of the next section.

There is one more process that potentially contributes to the monojet signature in the i2HDM, namely, $q \bar{q} \rightarrow h_{1} h_{2}+$ $g\left(g q \rightarrow h_{1} h_{2}+q\right)$, which we will refer to as the $h_{1} h_{2} j$ process. Feynman diagrams for this process are presented in Fig. 6. This process contributes to the monojet signature when the mass splitting between $h_{1}$ and $h_{2}$ is small, of the order of few giga-electron-volts. In this case, $h_{2}$ will decay to $h_{1}$ and soft jets or leptons from a virtual $Z$ which escapes detection. In spite of the fact that there is one mediator for this process, i.e., the $Z$ boson, one can see that $t$ - and $s$ channel topologies with a light quark in the propagator make this process different from simplified models with fermionic DM and a vector mediator, which have been studied so far in the literature, so it is worth exploring it in detail.

The parameter space for this process is characterized by two variables, $M_{h_{1}}$ and $M_{h_{2}}$, which fix its cross section for a given collider energy. It is also convenient to use $\Delta M=M_{h_{2}}-M_{h_{1}}$, the mass difference between the two particles, instead of $M_{h_{2}}$. In Fig. 7, we present the cross section for the $h_{1} h_{2} j$ process in the $\left(M_{h_{1}}, \Delta M\right)$ plane. The cross section has been evaluated with an initial cut, $p_{\mathrm{T}}^{\text {jet }}>100 \mathrm{GeV}$. One can see that, in this plane, the pattern of the cross section isolevels takes a simple form. One can also note that in case of $M_{h_{1}} \simeq 50-60 \mathrm{GeV}$ and small $\Delta M$ the cross section is of the order of $100 \mathrm{fb}$, which could be in the region of the LHC sensitivity. This cross section is comparable to that of the $h_{1} h_{1} j$ process for $\lambda_{345}=0.01$ and the same mass, which makes this kind of process important for the parameter space region where $\lambda_{345}$ is small. It is important to stress that the cross section for the $h_{1} h_{2} j$ process is independent of $\lambda_{345}$; therefore, this process

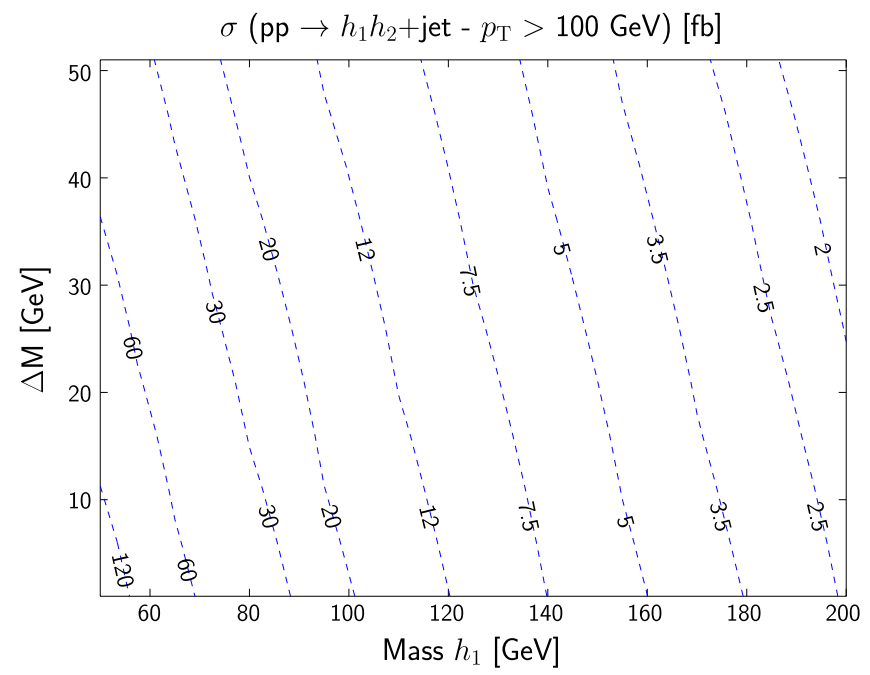

FIG. 7. Cross section vs DM mass $M_{h_{1}}$ and $\Delta M=M_{h_{2}}-M_{h_{1}}$ for the monojet process $h_{1} h_{2} j$ at the LHC@13 TeV. This process gives rise to a monojet signal if the mass difference $\Delta M$ is small enough, such that the decay $h_{2} \rightarrow h_{1}+X$ gives rise only to $E_{\mathrm{T}}^{\mathrm{miss}}+$ soft undetected leptons or jets. Here, the cross section was evaluated for the initial cut $p_{\mathrm{T}}^{\mathrm{jet}}>100 \mathrm{GeV}$. 


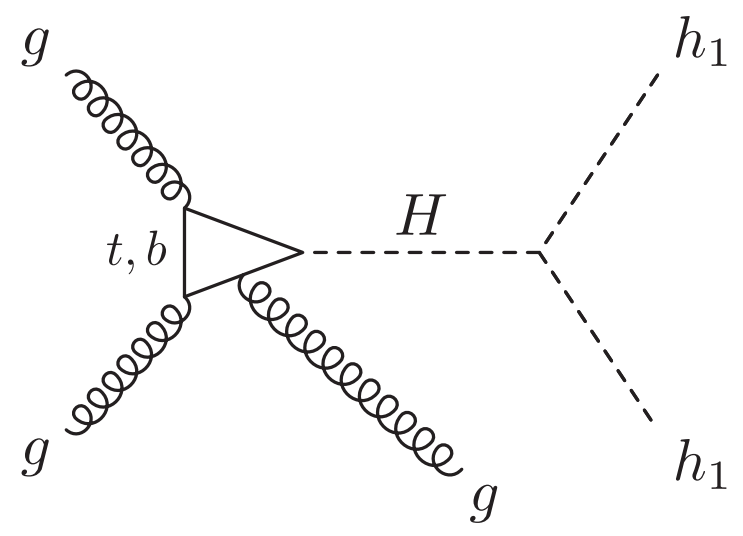

FIG. 8. Representative Feynman diagram for the one-loop (topand bottom-quark) induced $g g \rightarrow h_{1} h_{1} g$ process under study.

would provide a probe of the i2HDM parameter space, which is complementary to the $h_{1} h_{1} j$ process. $^{3}$

\section{A. Validity of the effective $g g H$ vertex approach}

The SM $g g H$ vertex is dominantly generated by the topquark loop (with a small bottom-quark contribution). It is known that integrating out the top quark is a good approximation for Higgs production processes when considering inclusive rates, as long as the Higgs boson is not far off shell or with high transverse momentum. The literature on this subject is vast, and we refer the reader to the corresponding sections in Ref. [59] and references therein. In case of our study, however, the selection of large transverse momentum of the jet (done to increase the signal-to-background ratio), which is typically bigger then the top-quark mass, is likely to lead to the breakdown of the heavy top-quark approximation.

From one of the representative one-loop diagrams presented in Fig. 8 for the $g g \rightarrow h_{1} h_{1} g$ process, one can see that a high $p_{T}$ jet emitted from the top-quark loop can "resolve" the top quark in the loop if the transverse momentum of the jet is large enough. This effect is crucial since the monojet $p_{T}$ and $E_{\mathrm{T}}^{\text {miss }}$ distributions from the EFT approximation (which one could be tempted to use for the sake of simplicity) could be different from those described by the exact loop calculation. This is even more crucial for us, due to the $E_{\mathrm{T}}^{\text {miss }}$ shape-analysis techniques which we use in our study. Therefore, we have compared the $E_{\mathrm{T}}^{\text {miss }}$ shapes for the events simulated using the EFT heavy top-quark

\footnotetext{
${ }^{3}$ On similar footing, we should finally mention that the monojet signature emerging from the i2HDM also sees a component in which an $h_{2}$ pair is produced, when $\Delta M$ is very small. The production topologies of this process, henceforth $h_{2} h_{2} j$, are the same as those for the $h_{1} h_{1} j$ case, though the yield is generally smaller. We nonetheless include this channel in our simulations, yet we will not dwell on it separately.
}

approximation to those from the exact one-loop calculation. For this purpose, we have simulated the process of Higgs boson production in association with a jet and scanned over the mass of the Higgs boson, corresponding to the different invariant masses of the DM pair.

For this specific study, our simulations have been performed with MADGRAPH5 $[60,61]$ using the NNPDF2.3 PDF set [58]. We have compared results for two models: MADGRAPH5 native SM implementation with the effective $g g H$ vertex and the SM at one-loop implementation. Using this setup, we have scanned over a range of Higgs boson masses and compared the Higgs boson $p_{T}$ and $\eta$ distributions for the effective $g g H$ vertex and one-loop level implementations. The results presented in Fig. 9 are evaluated for different benchmarks, corresponding to different Higgs masses (for both effective vertex and one-loop simulations) and with contributions of either top or bottom quarks, or both. We have applied an initial cut $p_{\mathrm{T}}^{\text {jet }}>$ $200 \mathrm{GeV}$ for this study. The differences between effective vertex and one-loop distributions are quite large for large transverse momenta, and the role of the bottom quark in the loop is - as one may expect-rather marginal. The pseudorapidity distribution is not affected as much. It is, however, interesting to notice that larger invariant masses shift the distribution from a central-peaked shape to a more forward-backward behavior. For a sanity check, we have also evaluated the effect of setting the top-quark mass to $10 \mathrm{TeV}$ in the one-loop calculation, so that it can be effectively cross-checked with the effective vertex results, and as one can see, indeed it agrees with those.

As a result of this comparison, we have defined a $k$-factor,

$$
k_{f}=\frac{\sigma\left(p p \rightarrow h_{1} h_{1} j\right)_{\mathrm{one}-\mathrm{loop}}}{\sigma\left(p p \rightarrow h_{1} h_{1} j\right)_{\mathrm{EFT}}},
$$

which provides correspondence between the effective vertex and one-loop results in the $p_{T}$ distribution of the Higgs boson as a function of two variables: $E_{\mathrm{T}}^{\text {miss }}$ and $M_{\text {(DM,DM) }}$ (the invariant mass of the DM pair). This $k$-factor is pictorially presented in Fig. 10. One can see that, for large $E_{\mathrm{T}}^{\text {miss }}$ values, the effect of the breakdown of the effective vertex approximation is dramatic. For example, for $E_{\mathrm{T}}^{\text {miss }}=1 \mathrm{TeV}$, the effective vertex approximation overestimates the one-loop result by 1 order of magnitude, i.e., $k_{f} \simeq 0.1$. At the same time, for smaller values of $E_{\mathrm{T}}^{\text {miss }}$, the effective vertex approximation can even underestimate the one-loop result, which happens for large values of the $M_{\text {(DM,DM) }}$ invariant mass of the DM pair; for example, for $E_{\mathrm{T}}^{\text {miss }}=300 \mathrm{GeV}$ and $M_{(\mathrm{DM}, \mathrm{DM})}=500 \mathrm{GeV}$, one finds $k_{f} \simeq 1.5$.

It is finally very important to stress that the $k$-factor which was found in two recent studies at next-to-leading order in QCD $[62,63]$ is very close (within few percent) to 

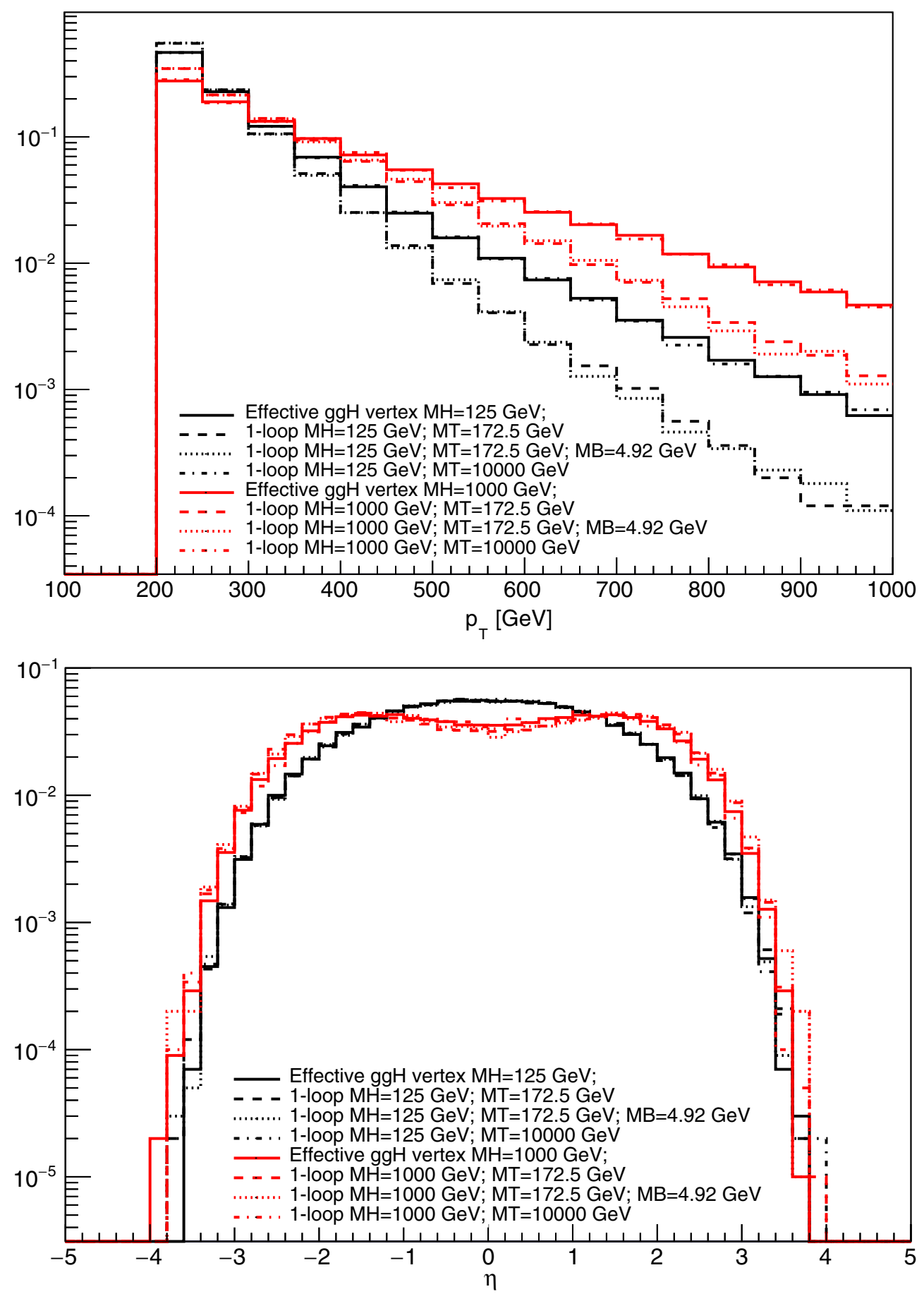

FIG. 9. Shape of transverse momentum (top panel) and pseudorapidity (bottom panel) distributions for a Higgs boson produced in association with one jet. Solid curves correspond to the distributions for the effective $g g H$ vertex approximation, dashed ones are for the one-loop result with only the top quark in the loop, dotted ones are for the one-loop result with both top and bottom quarks in the loop, and dot-dashed ones are for a very heavy quark in the loop $\left(m_{\mathrm{t}}=10 \mathrm{TeV}\right)$ for the purpose of cross-checking the effective $g g H$ vertex approximation. Black and red colors correspond to $M_{(\mathrm{DM}, \mathrm{DM})}=125$ and $1000 \mathrm{GeV}$, respectively.

the one we have found here at the leading order (LO) only (see also Ref. [64] where similar work is presented). Hence, based on our findings in this section, for our analysis below, we use one-loop results (i.e., at LO in QCD) and take into account the contributions from both top and bottom quarks.

\section{B. LHC potential to probe the i2HDM parameter space}

\section{Benchmarks}

Taking into account all the constraints, and especially the recent XENON1T ones, we suggest a set of six benchmark 


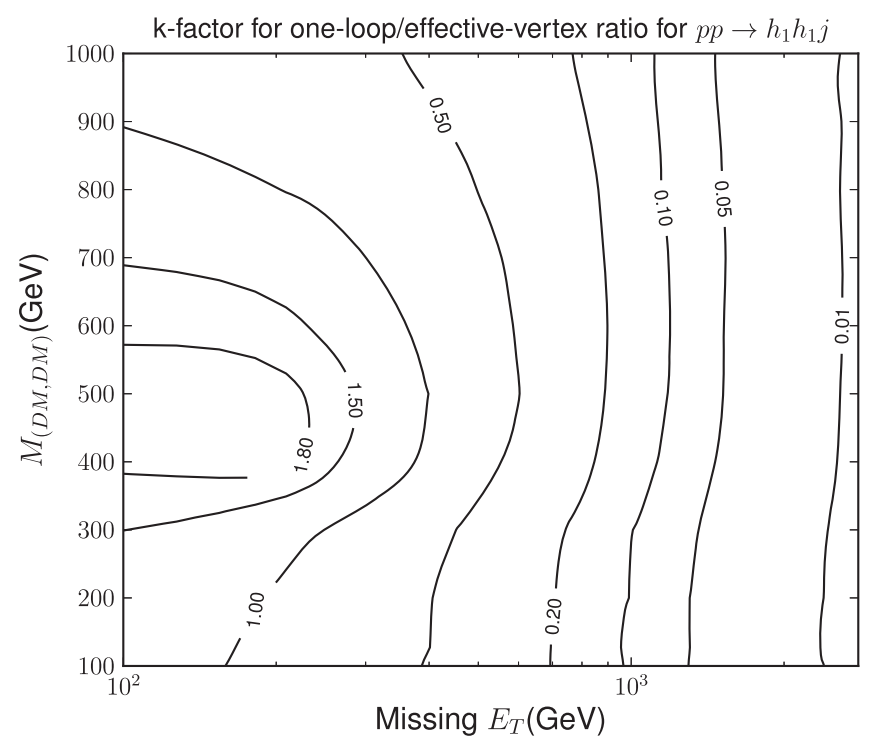

FIG. 10. The $k$-factor $k_{f}$ defined in the text as a function of the $E_{\mathrm{T}}^{\mathrm{miss}}$ and invariant mass of the DM pair. The $k$-factor values are indicated in the contour lines.

(BM) points, BM1 to BM6, summarized in Table I and described below:

(i) BM1: Both $M_{h_{1}}$ and $M_{h_{2}}$ are below $M_{\mathrm{H}} / 2$ contributing to about $20 \%$ of the invisible Higgs boson decay and yielding about $800 \mathrm{fb}$ of cross section for the monojet signature (which is high enough to be tested at the HL-LHC as we will discuss below) coming from the cumulative sum of the $h_{1} h_{1} j$, $h_{1} h_{2} j$, and $h_{2} h_{2} j$ processes. To measure the XENON1T sensitivity, we use the SI DM scattering rate on the proton $\left(\sigma_{\mathrm{SI}}^{p}\right)$ accompanied by its ratio to the experimental limit from XENON1T, following rescaling with the relic density, $R_{\mathrm{SI}}^{\mathrm{XENON1T}}=$ $\left(\sigma_{\mathrm{SI}}^{p} / \sigma_{\mathrm{SI}}^{\mathrm{XENON1T}}\right) \cdot\left(\Omega_{\mathrm{DM}} / \Omega_{\mathrm{DM}}^{\text {Planck }}\right)$, which is equal to 0.29 for this benchmark, i.e., about a factor of 3 below the current XENON1T sensitivity. The DM relic density for this point is below the Planck constraints because of the $h_{1} h_{2}$ coannihilation.

(ii) $B M 2$ : Only $M_{h_{1}}$ is below $M_{\mathrm{H}} / 2$, and the value of $\lambda_{345}$ is chosen to be small enough for the DM relic density to match both the upper and lower Planck constraints. In this case, the invisible Higgs boson decay to DM is only $2 \%$, and the respective rate of the $h_{1} h_{1} j$ monojet signal is only $74.6 \mathrm{fb}$. This point, with $R_{\mathrm{SI}}^{\mathrm{XENON} 1 \mathrm{~T}}=0.75$, is likely to be tested with future DM DD experiments since its value is not far from the present XENON1T limit.

(iii) BM3: Only $M_{h_{1}}=60 \mathrm{GeV}$ is below $M_{\mathrm{H}} / 2$, but $M_{h_{2}}=68 \mathrm{GeV}$ is quite close to it. Because of the large invisible Higgs boson decay to DM with $\operatorname{Br}\left(H \rightarrow h_{1} h_{2}\right)=0.25 \%$, the leading signal at the LHC will be a monojet from $h_{1} h_{1} j$, with a rate above

TABLE I. BM points from the i2HDM parameter space together with corresponding observables: DM relic density $\left(\Omega_{\mathrm{DM}} h^{2}\right), \mathrm{SI}$ DM scattering rate on the proton $\left(\sigma_{\mathrm{SI}}^{p}\right)$ accompanied by its ratio to the experimental limit from XENON1T following rescaling with the relic density, $R_{\mathrm{SI}}^{\mathrm{XENON1T}}=\left(\sigma_{\mathrm{SI}}^{p} / \sigma_{\mathrm{SI}}^{\mathrm{XENON1T}}\right) \cdot\left(\Omega_{\mathrm{DM}} / \Omega_{\mathrm{DM}}^{\mathrm{Plank}}\right)$ plus the LHC cross sections at the LHC@13 TeV with a $p_{T}^{\text {jet }}>100 \mathrm{GeV}$ cut applied.

\begin{tabular}{|c|c|c|c|c|c|c|}
\hline $\mathrm{BM}$ & 1 & 2 & 3 & 4 & 5 & 6 \\
\hline$M_{h_{1}}(\mathrm{GeV})$ & 55 & 55 & 60 & 60 & 70 & 80 \\
\hline$M_{h_{2}}(\mathrm{GeV})$ & 62 & 110 & 68 & 68 & 78 & 81 \\
\hline$M_{h_{+}}(\mathrm{GeV})$ & 120 & 120 & 100 & 100 & 78 & 81 \\
\hline$\lambda_{345}$ & 0.01 & 0.0065 & 0.033 & 0.0001 & 0.01 & 0.0 \\
\hline$\lambda_{2}$ & 1.0 & 1.0 & 1.0 & 1.0 & 1.0 & 1.0 \\
\hline$\Gamma_{h_{2}}(\mathrm{GeV})$ & $1.307 \times 10^{-8}$ & $2.926 \times 10^{-4}$ & $2.564 \times 10^{-8}$ & $2.564 \times 10^{-8}$ & $2.627 \times 10^{-8}$ & $7.314 \times 10^{-13}$ \\
\hline$\Gamma_{h_{+}}(\mathrm{GeV})$ & $1.549 \times 10^{-3}$ & $9.905 \times 10^{-4}$ & $1.137 \times 10^{-4}$ & $1.137 \times 10^{-4}$ & $3.666 \times 10^{-8}$ & $7.587 \times 10^{-13}$ \\
\hline$\Omega_{\mathrm{DM}} h^{2}$ & $1.78 \times 10^{-2}$ & $1.10 \times 10^{-1}$ & $1.37 \times 10^{-4}$ & $1.04 \times 10^{-1}$ & $4.56 \times 10^{-2}$ & $7.52 \times 10^{-3}$ \\
\hline$\sigma_{\mathrm{SI}}^{p}(\mathrm{pb})$ & $1.75 \times 10^{-10}$ & $7.37 \times 10^{-11}$ & $1.59 \times 10^{-9}$ & $1.46 \times 10^{-14}$ & $1.07 \times 10^{-10}$ & 0.0 \\
\hline$R_{\mathrm{SI}}^{\mathrm{XENON1T}}$ & 0.29 & 0.75 & 0.020 & $1.4 \times 10^{-4}$ & 0.45 & 0.0 \\
\hline $\operatorname{Br}\left(H \rightarrow h_{1} h_{1}\right)$ & $4.15 \times 10^{-2}$ & 0.022 & 0.25 & $3.1 \times 10^{-6}$ & 0.0 & 0.0 \\
\hline $\operatorname{Br}\left(H \rightarrow h_{2} h_{2}\right)$ & $1.59 \times 10^{-1}$ & 0.0 & 0.0 & 0.0 & 0.0 & 0.0 \\
\hline \multicolumn{7}{|l|}{$\sigma_{\mathrm{LHC} @ 13 \mathrm{TeV}}(\mathrm{fb})$} \\
\hline$h_{1} h_{1} j$ & $1.46 \times 10^{2}$ & 74.6 & 857 & $1.08 \times 10^{-2}$ & $4.96 \times 10^{-3}$ & 0.0 \\
\hline$h_{2} h_{2} j$ & $5.47 \times 10^{2}$ & $3.88 \times 10^{-1}$ & $3.06 \times 10^{-1}$ & $8.00 \times 10^{-2}$ & $5.50 \times 10^{-2}$ & $5.34 \times 10^{-4}$ \\
\hline$h_{1} h_{2} j$ & $1.04 \times 10^{2}$ & 34.1 & 77.4 & 77.0 & 49.6 & 39.0 \\
\hline$h_{1} h^{ \pm} j$ & 49.2 & 49.0 & 65.0 & 65.5 & 83.9 & 66.5 \\
\hline$h_{2} h^{ \pm} j$ & 44.9 & 24.7 & 58.0 & 57.9 & 72.1 & 65.4 \\
\hline$h^{ \pm} h^{ \pm} j$ & 13.0 & 13.0 & 20.9 & 16.2 & 39.2 & 35.3 \\
\hline
\end{tabular}


$800 \mathrm{fb}$, complemented by the $h_{1} h_{2} j$ process with rate $77.4 \mathrm{fb}$, which are high enough to be tested at the HL-LHC.

(iv) BM4: $M_{h_{1}}=60 \mathrm{GeV}$ and $M_{h_{2}}=68 \mathrm{GeV}$ as in BM3, but $\lambda_{345}$ is chosen to be low enough such that the DM relic density, governed by $h_{1} h_{2}$ coannihilation, is within the upper and lower Planck constraints. This point is unlikely to be tested by DD $\mathrm{DM}$ experiments in the near future, while the LHC could potentially test it shortly via a combination of $h_{1} h_{2} j, h_{1} h^{ \pm} j, h_{2} h^{ \pm} j$, and $h^{ \pm} h^{ \pm} j$ signatures, which are outside the scope of this paper.

(v) BM5: With all inert scalars close in mass, $M_{h_{1}}=70 \mathrm{GeV}, M_{h_{2}}=78 \mathrm{GeV}, M_{h^{ \pm}}=78 \mathrm{GeV}$, so all $h_{1} h_{2} j, h_{1} h^{ \pm} j, h_{2} h^{ \pm} j$, and $h^{ \pm} h^{ \pm} j$ channels contribute to the monojet signature (since both $h_{2}$ and $h^{ \pm}$promptly decay to $h_{1}$ and soft leptons escaping detection) with a total rate of about $250 \mathrm{fb}$, which is close to the exclusion limit at the HL-LHC as we will see below.

(vi) BM6: Masses for all inert scalars are even closer to each other in comparison to BM5, as $M_{h_{1}}=80 \mathrm{GeV}$, $M_{h_{2}}=81 \mathrm{GeV}$ and $M_{h^{ \pm}}=81 \mathrm{GeV}$. Since $\lambda_{345}=0$ the rates of $h_{1} h_{1} j$ and $h_{2} h_{2} j$ processes are vanishing. With this configuration all $h_{1} h_{2} j, h_{1} h^{ \pm} j, h_{2} h^{ \pm} j$, and $h^{ \pm} h^{ \pm} j$ channels contribute to the monojet signature with a total rate of about $210 \mathrm{fb}$, again close to the exclusion limit at the HL-LHC.

The masses of DM for BM1-BM6 were chosen below $100 \mathrm{GeV}$ in anticipation of the LHC sensitivity to the parameter space, which we present below. At present, the LHC experimental collaborations ATLAS and CMS do not have specific searches for the i2HDM; however, the results for generic DM searches in the jet $+E_{\mathrm{T}}^{\text {miss }}$ channel can be reinterpreted in the context of such a model. In order to compare the i2HDM to those limits, the following procedure is followed:

(i) The matrix elements that describe the hard interaction are simulated with CALCHEP, and event samples for different values of $M_{h_{1}}$ are produced. In order to concentrate on a region of phenomenological interest and simulate events with enhanced statistics, a lower threshold on the final state parton (either $q$ or $g$ ) is set at $p_{\mathrm{T}}>100 \mathrm{GeV}$. The event samples are produced in the Les Houches Event format for further processing.

(ii) In order to accurately describe the $p_{\mathrm{T}}$ distribution, each event is weighted with the $k$-factor estimated in the previous section, according to its parton $p_{\mathrm{T}}$ and the invariant mass of the DM-DM system.

(iii) Each event sample is then passed to PYTHIA8.2 $[65,66]$ for the proper treatment of parton showering, hadronization, and underlying event effects. The aforementioned NNPDF set is again deployed through the LHAPDF6 tool [67]. (iv) The DELPHES3 framework for fast simulation of generic collider experiments [68] is used to simulate the event reconstruction by the CMS experiment. Specifically, the detector parametrization for CMS described as standard in the card DELPHES_ CARD_CMS.TCL from the DELPHES distribution is used.

(v) A set of selection criteria is applied to the simulated reconstructed events. In the experimental collaborations, these criteria aim to reduce both the $S M$ backgrounds (mainly composed of inclusive $W / Z$ boson production) and instrumental noise that mimics the appearance of a single, highly energetic jet in the event. We disregard the effect of the latter phenomenon in our analysis, though.

After the fast detector level simulation described above, we performed an analysis of the missing transverse momentum distribution $E_{\mathrm{T}}^{\text {miss }}$ of the signal events. We compare the $E_{\mathrm{T}}^{\text {miss }}$ distribution predicted by a given signal sample to the standard model background prediction. That background prediction is a fundamental ingredient for our analysis; it can either be explicitly given by the experimental collaborations or estimated by an explicit calculation of the inclusive $W / Z$-boson production cross section shape. For each signal sample, we set upper limits on the production cross section of the monojet process. We compute the limits following an asymptotic approximation to the modified frequentist prescription known as the $\mathrm{CL}_{\mathrm{S}}$ technique $[69,70]$, in which systematic uncertainties are treated as nuisance parameters through use of the profile likelihood ratio. The only systematic uncertainty we consider in our analysis is the uncertainty in the background prediction. Throughout our study, the THETA framework [71] for modeling, and inference is used for all statistical analyses and limit-setting procedures.

We study the jet $+E_{\mathrm{T}}^{\text {miss }}$ signature from two signal processes, $h_{1} h_{1} j$ and $h_{1} h_{2} j$, in the presence of a small (a few $\mathrm{GeV}$ ) $M_{h_{2}}-M_{h_{1}}$ mass gap making $h_{1} h_{2} j$ to contribute to the monojet signature. In our study, we analyze $h_{1} h_{1} j$ and $h_{1} h_{2} j$ separately for two reasons: a) the rate of these processes depends upon different parameters, so they complement each other as i2HDM parameter space probes, and b) these processes have different shapes in the $E_{\mathrm{T}}^{\mathrm{miss}}$ distribution because of the different nature and mass of the mediators.

\section{Results from Run 2 data}

At the beginning of Run 2, the LHC@ $13 \mathrm{TeV}$ delivered a total integrated luminosity of $4.2 \mathrm{fb}^{-1}$. The CMS Collaboration released a public result where $2.3 \mathrm{fb}^{-1}$ of data were used to search for DM production in association with jets or hadronically decaying vector bosons [72]. Henceforth, we will refer to this result as the "CMS Run 2 analysis." Supplementary material—data and Monte Carlo 
TABLE II. Initial selection cuts for the CMS Run 2 monojet analysis at $\sqrt{s}=13 \mathrm{TeV}$ [72]. Jets considered for the jet multiplicity and angular configuration selections are required to have $p_{\mathrm{T}}^{\text {jet }}>30 \mathrm{GeV}$ and $\left|\eta^{\text {jet }}\right|<2.5$.

\begin{tabular}{ll}
\hline \hline Quantity & Selection \\
\hline Leading jet $p_{\mathrm{T}}$ & $>100 \mathrm{GeV}$ \\
Leading jet $|\eta|$ & $<2.5$ \\
$E_{\mathrm{T}}^{\text {miss }}$ & $>200 \mathrm{GeV}$ \\
$\Delta \phi\left(E_{\mathrm{T}}^{\text {miss }}\right.$, jet $\left._{1 . .4}\right)$ & $>0.5$ \\
\hline \hline
\end{tabular}

background distributions as well as their uncertaintieswere made available by the collaboration and used to set limits on the i2HDM. Table II summarizes the experimental selection used for the CMS result, while Table III presents the data used for our study at $13 \mathrm{TeV}$.

The main change for the Run 2 selection was the update of the angular discriminant to suppress QCD multijet contributions, whereas in Run 1 , a strict requirement was imposed on the jet multiplicity and leading jets azimuthal distance in Run 2 CMS opted instead for an overall requirement of azimuthal separation between the measured $E_{\mathrm{T}}^{\mathrm{miss}}$ and the four leading hadronic jets. The selection efficiency for both the $h_{1} h_{1} j$ and $h_{1} h_{2} j$ processes can be seen in Fig. 11 and is around 10\%-25\% for the former and $18 \%-40 \%$ for the latter. We can understand this difference by noticing that $h_{1} h_{2} j$ production is mediated by a $Z$ boson while $h_{1} h_{1} j$ production is mediated by the

TABLE III. SM background prediction and observed data for the CMS Run 2 monojet analysis at $\sqrt{s}=13 \mathrm{TeV}$ [72] as a function of the $E_{\mathrm{T}}^{\text {miss }}$ variable.

\begin{tabular}{lrr}
\hline \hline Bin range $(\mathrm{GeV})$ & SM background & Observed data \\
\hline $200-230$ & $28654 \pm 171$ & 28601 \\
$230-260$ & $14675 \pm 97$ & 14756 \\
$260-290$ & $7666 \pm 68$ & 7770 \\
$290-320$ & $4215 \pm 48$ & 4195 \\
$320-350$ & $2407 \pm 37$ & 2364 \\
$350-390$ & $1826 \pm 32$ & 1875 \\
$390-430$ & $998 \pm 23$ & 1006 \\
$430-470$ & $574 \pm 17$ & 543 \\
$470-510$ & $344 \pm 12$ & 349 \\
$510-550$ & $219 \pm 9$ & 216 \\
$550-590$ & $134 \pm 7$ & 142 \\
$590-640$ & $98.5 \pm 5.8$ & 111 \\
$640-690$ & $58.0 \pm 4.1$ & 61 \\
$690-740$ & $35.2 \pm 2.9$ & 32 \\
$740-790$ & $27.7 \pm 2.7$ & 28 \\
$790-840$ & $16.8 \pm 2.2$ & 14 \\
$840-900$ & $12.0 \pm 1.6$ & 13 \\
$900-960$ & $6.9 \pm 1.2$ & 7 \\
$960-1020$ & $4.5 \pm 1.0$ & 3 \\
$1020-1160$ & $3.2 \pm 0.9$ & 1 \\
$1160-1250$ & $2.2 \pm 0.7$ & 2 \\
$1250-$ inf & $1.6 \pm 0.6$ & 3 \\
\hline \hline
\end{tabular}

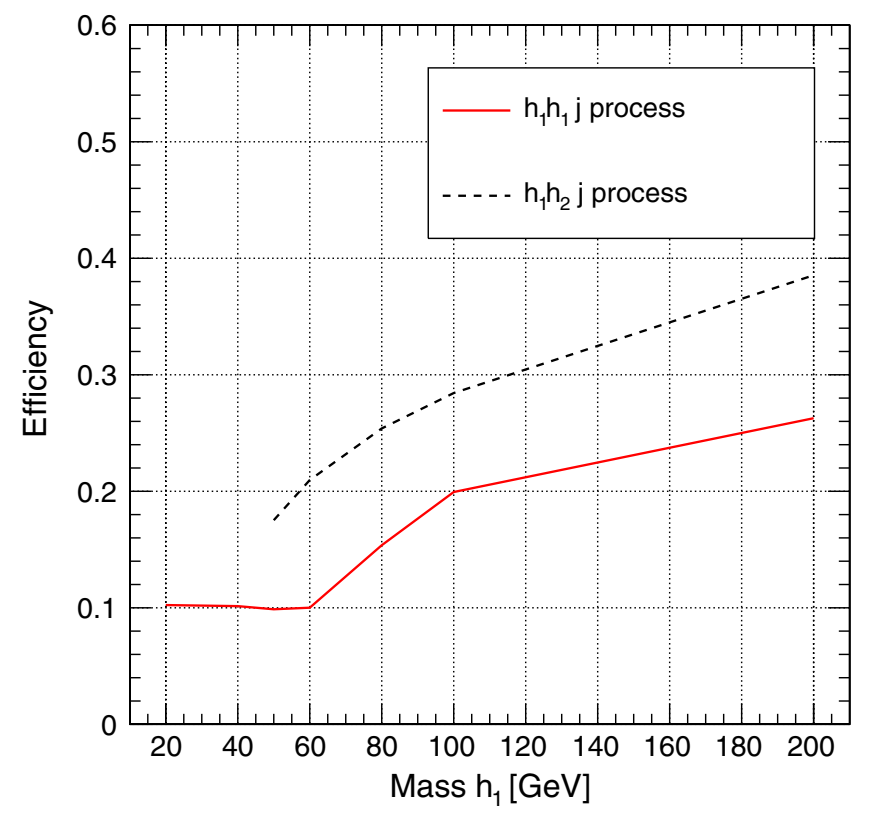

FIG. 11. Efficiency of the selection criteria described in Table II, on the simulated reconstructed events, for $h_{1} h_{1} j$ (solid red line) and $h_{1} h_{2} j$ (dashed black line). Here, $\sqrt{s}=13 \mathrm{TeV}$.

SM-like Higgs boson, which leads to a different $E_{\mathrm{T}}^{\text {miss }}$ spectrum. Figure 12 presents the comparison of the $E_{\mathrm{T}}^{\text {miss }}$ distributions for different DM masses for the signal from the $h_{1} h_{1} j$ (left panel) and $h_{1} h_{2} j$ (right panel) processes as well as for the background. One can notice that the $E_{\mathrm{T}}^{\text {miss }}$ distribution for the $h_{1} h_{2} j$ signal is indeed harder than the one for the $h_{1} h_{1} j$ case. This difference in $E_{\mathrm{T}}^{\text {miss }}$ shapes is related to the difference in the invariant mass of DM pair distributions, for $h_{1} h_{2} j$ and $h_{1} h_{1} j$ signals; as discussed in Ref. [13], a scalar mediator defines a softer invariant mass of the DM pair than a vector mediator (for similar masses), while the invariant mass of the DM pair in its turn is correlated with the shape of the $E_{\mathrm{T}}^{\text {miss }}$ distribution.

It can be observed from Fig. 12 that the $E_{\mathrm{T}}^{\text {miss }}$ spectrum of the signal is harder than that of the background for the whole range of DM masses sampled, especially for the large values, which agrees with the findings of Ref. [13], where it was shown that distributions at larger values of $M_{(\mathrm{DM}, \mathrm{DM})}$ have a flatter $E_{\mathrm{T}}^{\text {miss }}$ shape. Eventually, for higher values of $M_{h_{1}}, M_{\text {(DM,DM) }}$ will also be higher. This suggests two strategies for the signal and background comparison. A simpler analysis would be a so-called counting experiment, where a lower $E_{\mathrm{T}}^{\text {miss }}$ threshold ("cut") is defined and the spectrum is integrated above that value. This procedure produces a single event yield (with uncertainty) for both signal $\left(N_{\text {sig }}\right)$ and background $\left(N_{\text {bkg }}\right)$, and in the case of an observed limit, the total number of observed events $\left(N_{\text {obs }}\right)$ would also be available. Those are input to the limit-setting technique described in the previous section, through a single likelihood $\mathcal{L}\left(N_{\text {sig }}, N_{\text {bkg }}, N_{\text {obs }}\right)$. A more sophisticated 

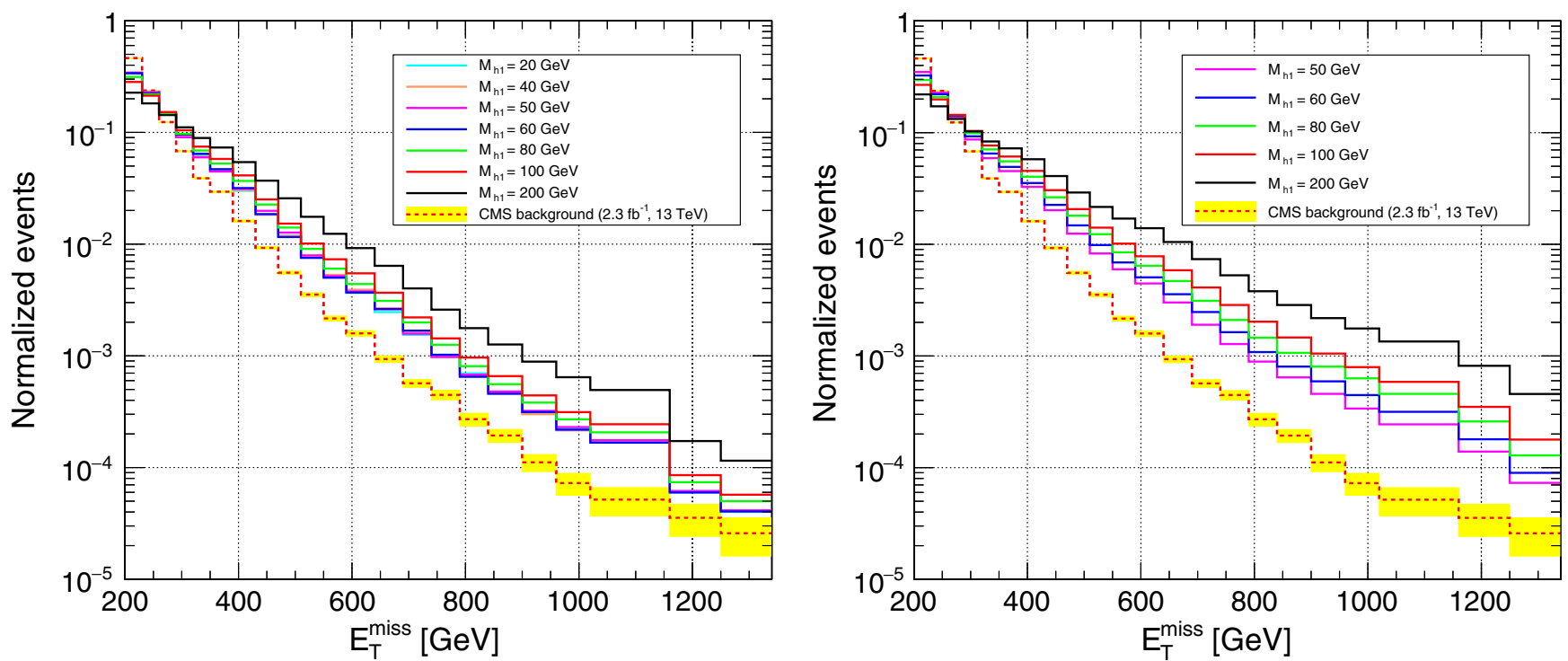

FIG. 12. Comparison of $E_{\mathrm{T}}^{\text {miss }}$ distributions between signals, for $h_{1} h_{1} j$ (left) and $h_{1} h_{2} j$ (right), for various DM masses, alongside the estimated (by CMS) experimental background for $\sqrt{s}=13 \mathrm{TeV}$.

analysis, in contrast, could take into account the coherent enhancement over all the $E_{\mathrm{T}}^{\text {miss }}$ spectrum that the presence of a signal would entail. In this strategy, the binned likelihood is written as the product of the single likelihoods of each bin over the relevant $E_{\mathrm{T}}^{\text {miss }}$ range. This will be called the shape analysis strategy. The CMS Run 1 analysis [73] used a series of counting experiments with different $E_{\mathrm{T}}^{\text {miss }}$ ranges, while the CMS Run 2 analysis employs a shape analysis. Figure 13 shows, for our signal samples and the background estimates from the CMS Run 2 analysis, the difference among four different analysis strategies: three counting experiments, with respective $E_{\mathrm{T}}^{\text {miss }}$ cuts of 200 , 470 , and $690 \mathrm{GeV}$, and a shape analysis with a lower threshold of $200 \mathrm{GeV}$. One can see that higher $E_{\mathrm{T}}^{\text {miss }}$ thresholds in the counting experiment make the expected limit become worse, while the shape analysis is able to leverage the coherent enhancements in all bins of $E_{\mathrm{T}}^{\text {miss }}$ that arises from the signal presence to set a better limit, an order of $30 \%$ improvement. We will therefore adopt the shape analysis strategy for the rest of this study.

Figure 14 shows the 95\% confidence level (CL) expected and observed exclusion limits as a function of $M_{h_{1}}$ derived using the CMS $13 \mathrm{TeV}$ background prediction and observed data with the Run 2 selection as described in Table II. In the left panel, we show the limit for the $h_{1} h_{1} j$ process. In order to compare with the actual signal rate, we show two signal lines for different values of $\lambda_{345}$. A red solid line presents the i2HDM cross section for $\lambda_{345}=0.019$ that is near the maximum allowed by the Higgs invisible decay search, when $M_{h_{1}}<M_{\mathrm{H}} / 2$. In this region, the SM-like Higgs boson is produced on shell, which enhances substantially the production cross section, and we can further notice a steep drop of the latter for $M_{h_{1}}>60 \mathrm{GeV}$. In contrast, for
$M_{h_{1}}>M_{\mathrm{H}} / 2$, there is no bound on $\lambda_{345}$ from the Higgs invisible decay width, and the cross section scales with $\lambda_{345}$ squared. We show with a blue dashed line the expected i2HDM cross section for maximally allowed $\lambda_{345}$ by the present data; it reaches 1.6 times the value that is around the

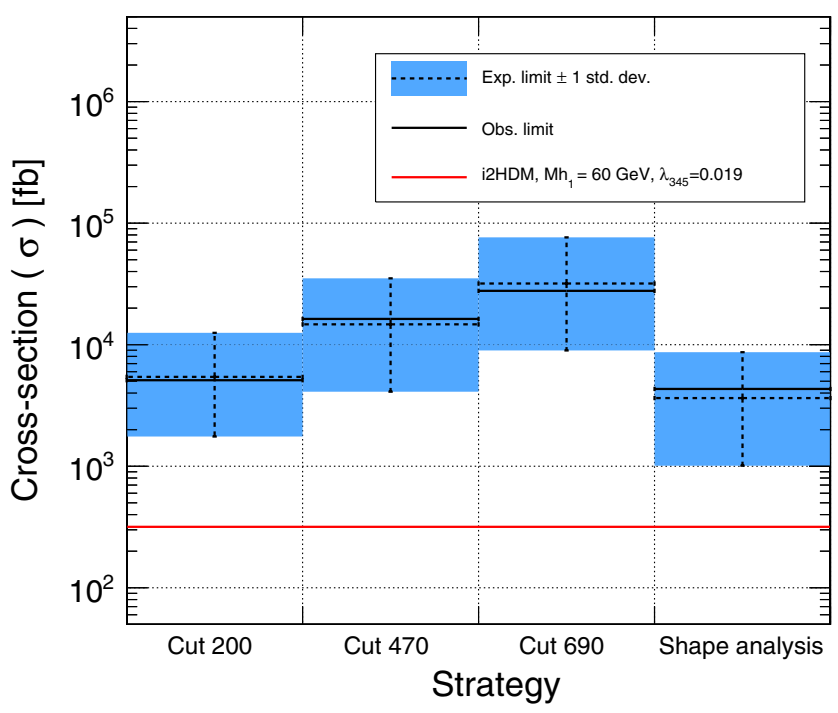

FIG. 13. Expected and observed limits for four different analysis strategies: three counting experiments, with lower thresholds of 200, 470 and $690 \mathrm{GeV}$, and a shape analysis with a lower threshold of $200 \mathrm{GeV}$. The counting experiments are able to set expected limits of $5.44 \mathrm{pb}, 14.7 \mathrm{pb}$, and $31.9 \mathrm{pb}$, respectively, while the shape analysis is able to set an expected limit of $3.64 \mathrm{pb}$, a $30 \%$ improvement over the counting experiment with lowest threshold. The red line is for the $h_{1} h_{1} j$ process with $M_{h_{1}}=60 \mathrm{GeV}, M_{h_{2}}=200 \mathrm{GeV}, \lambda_{345}=0.019$, giving a cross section of $0.317 \mathrm{pb}$. 

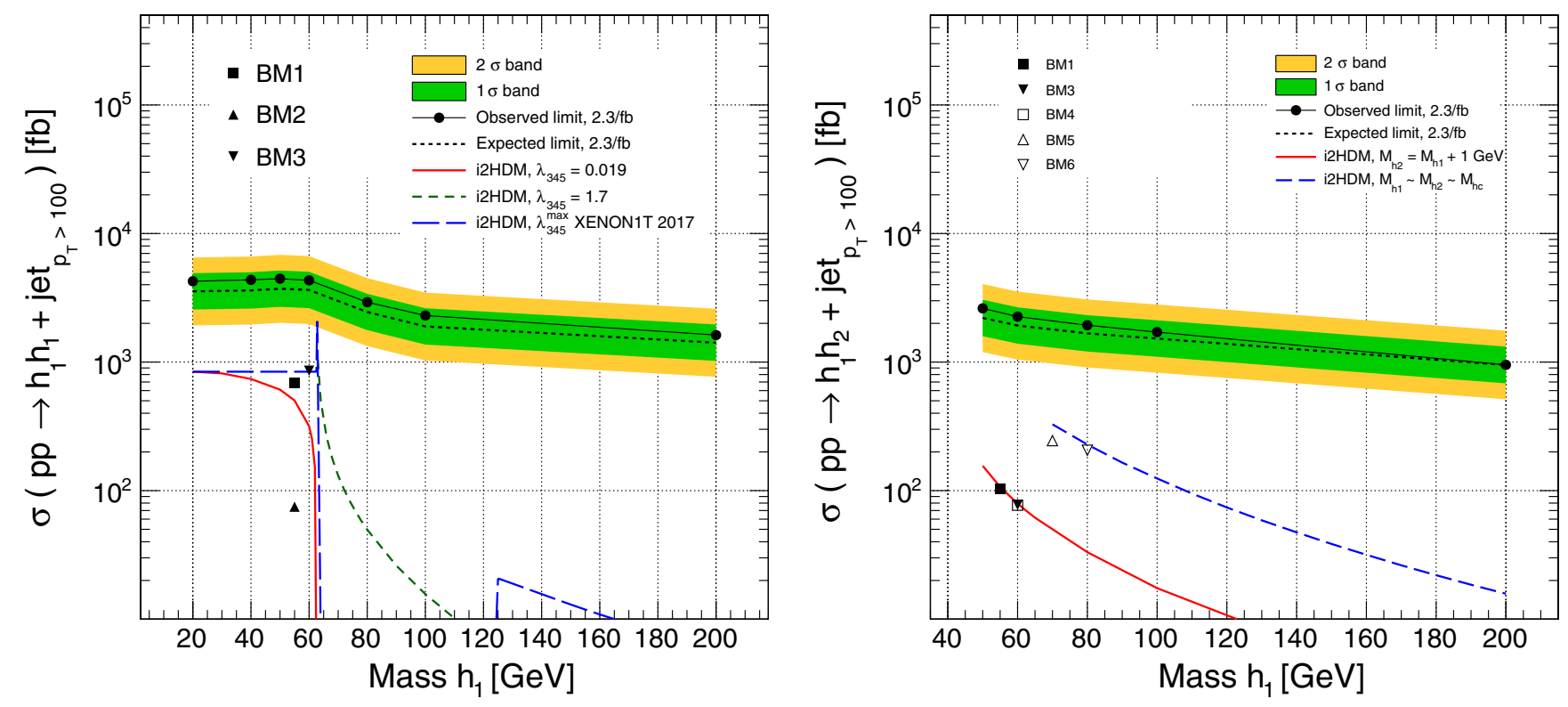

FIG. 14. Left: Expected and observed limits on the $h_{1} h_{1} j$ process for $2.3 \mathrm{fb}^{-1}$ of $13 \mathrm{TeV} p p$ collision data. The red solid line is the cross section for the parameter set $\lambda_{345}=0.019, M_{h_{2}}=200 \mathrm{GeV}$ while the green short dashed line is the cross section for the parameter set $\lambda_{345}=1.7, M_{h_{2}}=200 \mathrm{GeV}$. The blue dashed line is the combined contribution $h_{1} h_{1} j+h_{1} h_{2} j$ that is still allowed by XENON1T data (see text). Right: Expected and observed limits on the $h_{1} h_{2} j$ process for $2.3 \mathrm{fb}^{-1}$ of $13 \mathrm{TeV} p p$ collision data. The red solid line is the cross section for $M_{h_{2}}=M_{h_{1}}+1 \mathrm{GeV}$. The blue short dashed line is the cross section for a full degeneracy between $h_{1}, h_{2}$ and $h_{c}$, where additional processes involving the charged scalar could mimic the $h_{1} h_{2} j$ process. The cross section is plotted for values of $M_{h_{1}}$ larger than $\sim 70 \mathrm{GeV}$ to comply with the LEP bound on the charged Higgs mass [45]. In all cases the isolated symbols represent the benchmark points discussed in Table I. All cross sections are given for a $p_{\mathrm{T}}^{\text {jet }}>100 \mathrm{GeV}$ requirement.

maximum for $M_{h_{1}} \simeq M_{\mathrm{H}} / 2$ allowed by vacuum stability. Outside of the $M_{h_{1}} \simeq M_{\mathrm{H}} / 2$ region, $\lambda_{345}$ is strongly excluded by XENON1T data; e.g., in the interval $65 \mathrm{GeV}<M_{h_{1}}<70 \mathrm{GeV}$, the only values of $\lambda_{345} \lesssim$ 0.025 are allowed. We can see that, for the $2.3 \mathrm{fb}^{-1}$ data set, we exclude the $h_{1} h_{1} j$ process cross sections in the range of 4.3-1.6 pb for $M_{h_{1}}$ in the range 20-200 GeV, which does not exclude the i2HDM even for the highest allowed value of $\lambda_{345}$. For the $h_{1} h_{2} j$ process, we exclude cross sections in the range of $2.6-0.95 \mathrm{pb}$ for $M_{h_{1}}$ in the range $50-200 \mathrm{GeV}$, also not enough to set relevant limits on the i2HDM.

When $M_{h_{1}} \simeq M_{h_{2}}, h_{2}$ will decay to $h_{1}$ plus very soft products; thus, $h_{1} h_{1} j, h_{2} h_{2} j$, and $h_{1} h_{2} j$ production will contribute to the jet $+E_{\mathrm{T}}^{\text {miss }}$ signature. The $h_{2} h_{2} j$ and $h_{1} h_{1} j$ channels proceed via the same mediator and can be combined since they have the same $E_{\mathrm{T}}^{\text {miss }}$ shape (for small values of $\Delta M=M_{h_{2}}-M_{h_{1}}$ ). We indicate the predicted combined $h_{1} h_{1} j$ and $h_{2} h_{2} j$ cross section by the purple dashed line for $\lambda_{345}^{\max }$ in Fig. 14 (left panel). One can see that $2.3 \mathrm{fb}^{-1}$ monojet data are not quite sensitive even to the combined $h_{1} h_{1} j$ and $h_{2} h_{2} j$ signal at $\lambda_{345}^{\max }$.

As mentioned above, the $h_{1} h_{2} j$ production is mediated by $Z$-boson exchange (see Fig. 6) and has therefore a different $E_{\mathrm{T}}^{\mathrm{miss}}$, so we investigate it separately. The right panel of Fig. 14 presents the limit for the $h_{1} h_{2} j$ production process (for $\Delta M=1 \mathrm{GeV}$ ) and indeed demonstrates that the cross section limit for this process is different from the $h_{1} h_{1} j$ one because of their different kinematics. This process does not depend on the $\lambda_{345}$ coupling, and thus the cross section is determined by the masses of $h_{1}$ and $h_{2}$; only, here, the expected signal rate represented by the red line indicates that it is well below the present limit.

\section{Projections for the HL-LHC}

For a next step in our study, we have found the projected LHC potential at higher integrated luminosities of 30, 300, and $3000 \mathrm{fb}^{-1}$ with the last value posited as the ultimate benchmark for the HL-LHC. For this study, we made the following simplifying assumptions:

(i) The SM background to the monojet searches at the HL-LHC is still going to be dominated by inclusive EW production of $W$ and $Z$ bosons, with strong production of $t \bar{t}$ pairs being a minor background.

(ii) The upgraded experiments will be successful in maintaining the physics performance demonstrated during Run 1 and Run 2, even in view of a much higher pileup in the range of $\langle P U\rangle=140-200$.

(iii) The change from 13 to $14 \mathrm{TeV}$ center-of-mass energy will not change the kinematic distribution of the reconstructed object in any significant way, neither for the SM background nor for the i2HDM processes. 

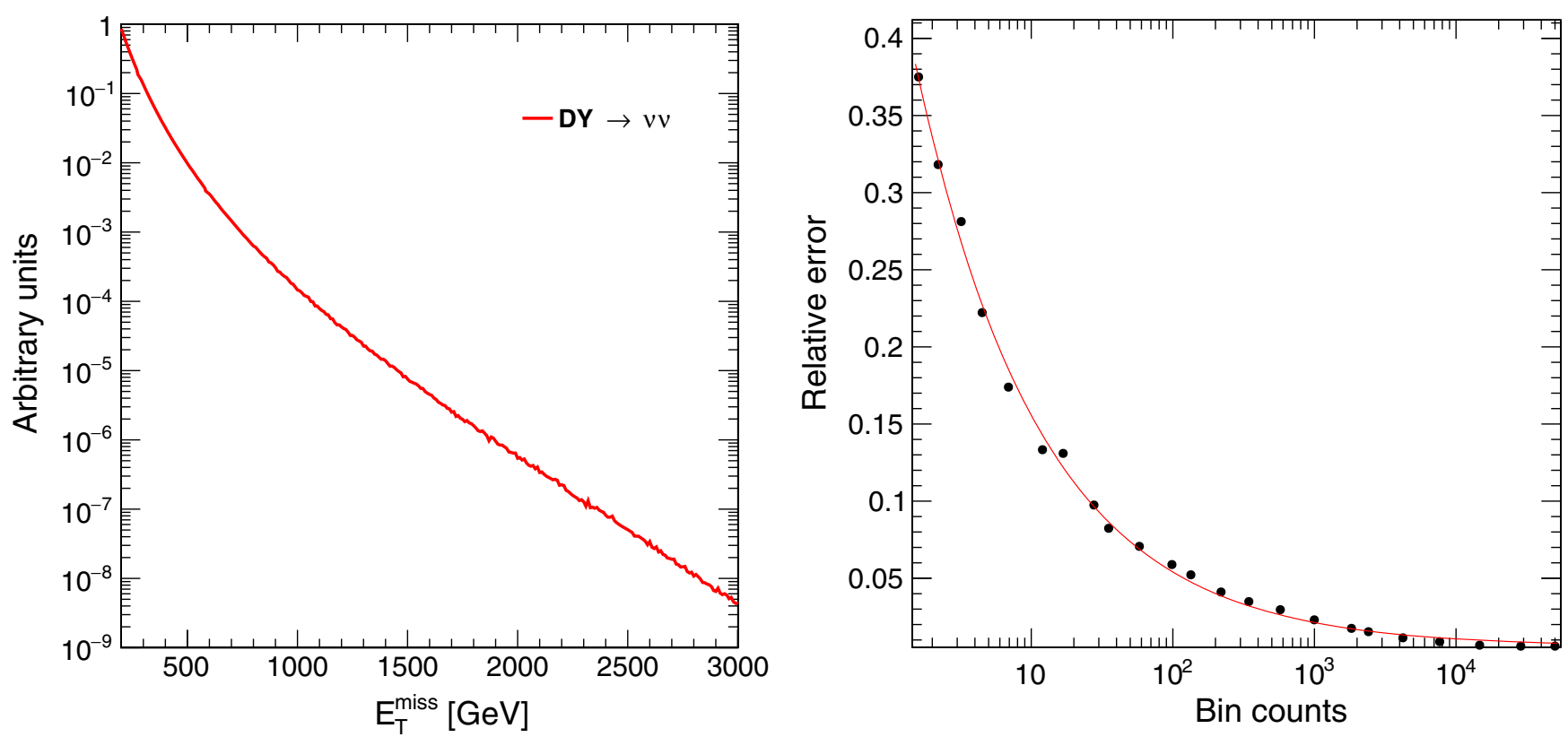

FIG. 15. Left: $E_{\mathrm{T}}^{\text {miss }}$ distribution from $p p \rightarrow Z \rightarrow \nu \bar{\nu} j$ process produced with CALCHEP, for $p p$ collisions at $\sqrt{s}=13$ TeV. Right: Relative error in the background estimate as a function of bin counts, as extracted from Table III.

(iv) The overall analysis strategy will be kept very similar to that in Table II. As such, shape, yield, and uncertainty of both the signal and background can be scaled to the desired luminosities.

While the extrapolation of the signal distributions to the HL-LHC is a simple rescaling, the estimate of the tails of the $W / Z$ inclusive $p_{\mathrm{T}}$ distributions is far from trivial. For the purposes of our study, we estimated the shape of the SM background directly from a simulation of $Z \rightarrow \nu \bar{\nu} j$ produced with CALCHEP, shown in the left panel of Fig. 15, while the normalization is approximated by a rescaling of the CMS results, since the efficiency of the selection is assumed to be the same. Since the background is primarily estimated from data distributions in control regions, we expect that the overall uncertainty in the $E_{\mathrm{T}}^{\text {miss }}$ prediction follows approximately a $1 / \sqrt{N}$ distribution. The right panel of Fig. 15 shows the relative errors in each bin from Table III as a function of the bin content. One can see that, indeed, it follows the aforementioned distribution, but in addition, it also has a constant term $(\sim 0.6 \%)$ that can be understood to represent uncertainties that are not statistical in nature. We use the following equation for our bin-by-bin error estimate,

$$
\sigma_{\text {bin }}^{(\text {rel })} \equiv \frac{\sigma_{\text {bin }}}{N_{\text {bin }}} \simeq \frac{0.46}{\sqrt{N_{\text {bin }}}}+0.6 \%
$$

where $N_{\text {bin }}$ and $\sigma_{\text {bin }}$ are the content and uncertainty of the given bin. The numerical values in Eq. (10) are obtained through a fit to the relative errors from Table III.
Our final background estimate for the extrapolation to the HL-LHC is therefore done through the following procedure:

(i) We find the shape of the $E_{\mathrm{T}}^{\text {miss }}$ distribution from the $p p \rightarrow Z \rightarrow \nu \bar{\nu} j$ process (Fig. 15).

(ii) We normalize the histogram such that the integral $I_{L}$ in the range $200-1250 \mathrm{GeV}$ is

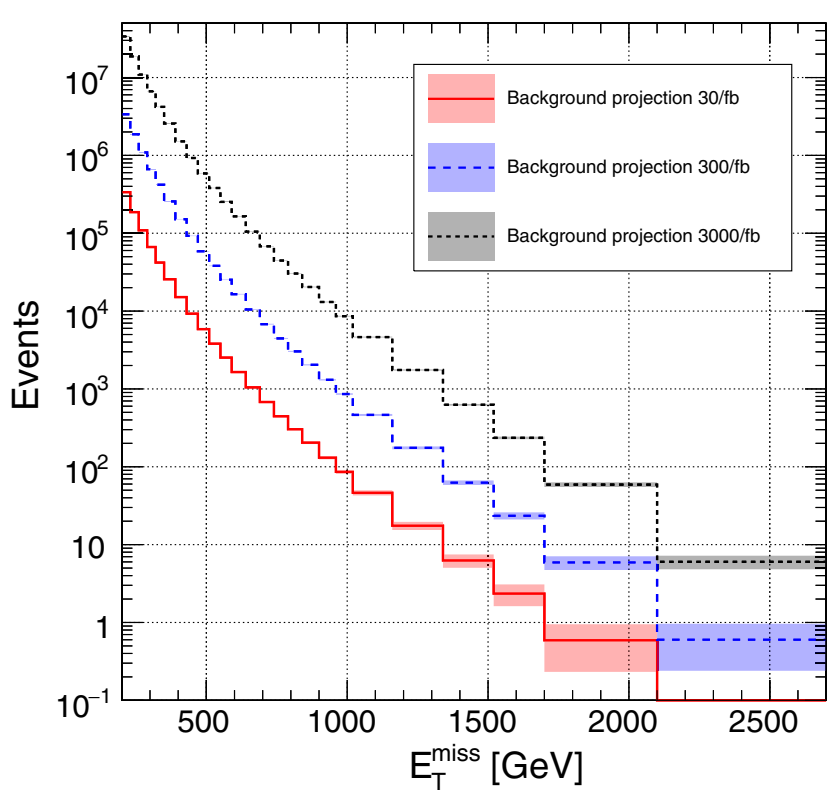

FIG. 16. Background extrapolation for 30 (red solid line), 300 (blue dashed line), and $3000 \mathrm{fb}^{-1}$ (black dotted line). The errors (shaded areas) are estimated through the procedure described in the text. 

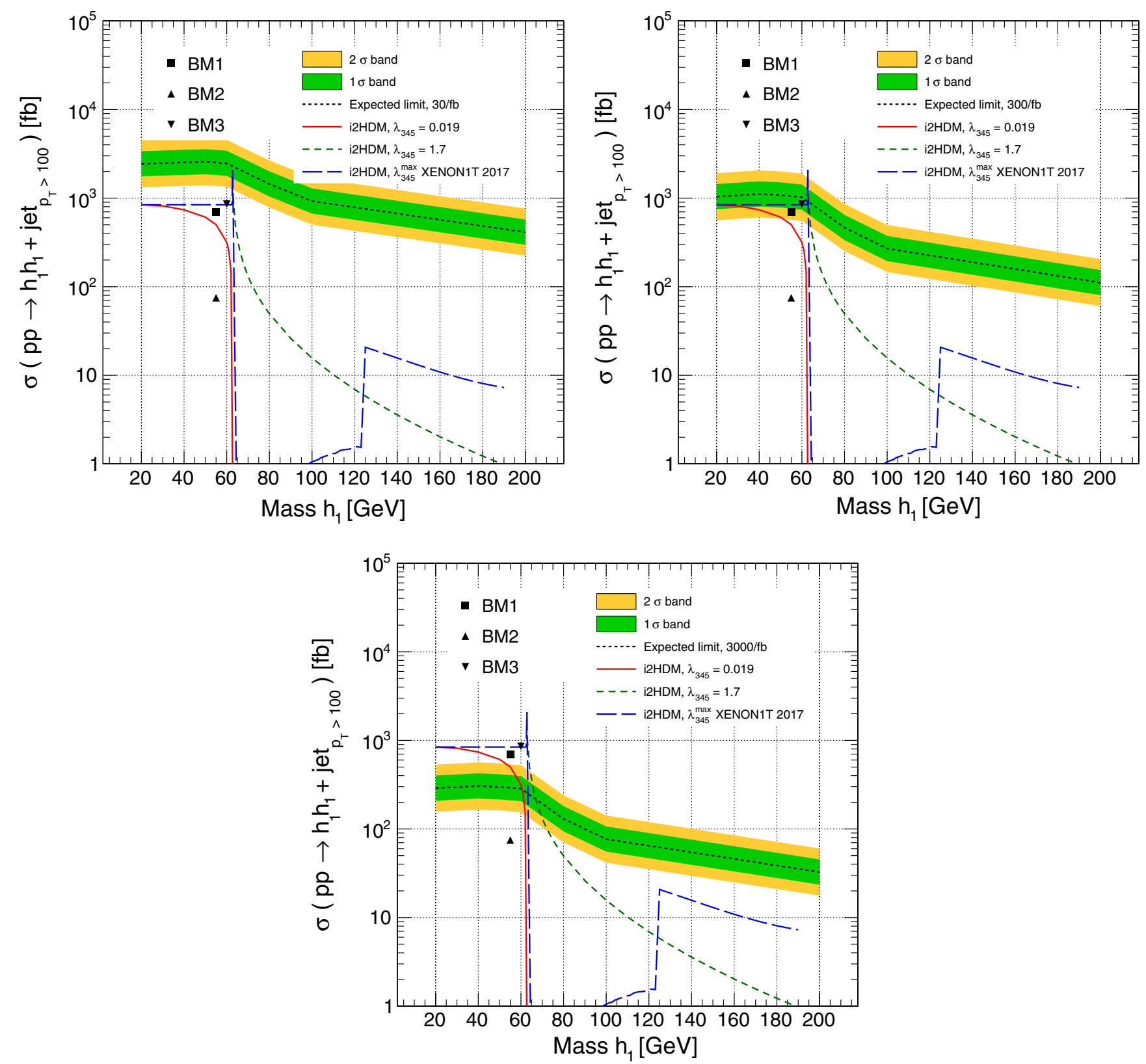

FIG. 17. Expected limits for the $h_{1} h_{1} j$ process for 30 (top left), 300 (top right), and $3000 \mathrm{fb}^{-1}$ (bottom). The red solid line is the cross section for the parameter set $\lambda_{345}=0.019, M_{h_{2}}=200 \mathrm{GeV}$, while the green short dashed line is the cross section for the parameter set $\lambda_{345}=1.7, M_{h_{2}}=200 \mathrm{GeV}$. The blue dashed line is the combined contribution $h_{1} h_{1} j+h_{1} h_{2} j$ for $M_{h_{2}}=M_{h_{1}}+1 \mathrm{GeV}$ and the maximal value of $\lambda_{345}^{\max }$ allowed by XENON1T data (see the text). The isolated symbols represent the benchmark points discussed in Table I. All cross sections are always given for a $p_{\mathrm{T}}^{\text {jet }}>100 \mathrm{GeV}$ requirement.

$$
I_{L}=\frac{L_{\text {target }}}{L_{2015}} \cdot N_{\text {events }}
$$

where $L_{\text {target }}$ is the target luminosity $(30,300$, or $\left.3000 \mathrm{fb}^{-1}\right), L_{2015}=2.3 \mathrm{fb}^{-1}$ is the integrated luminosity of Ref. [72], and $N_{\text {events }}=61978.6$ is the total number of events in the aforementioned range, from Table III. This normalization is produced to approximate the efficiency of the CMS selection on the real SM background.

(iii) We find the bin-by-bin errors according to the formula in Eq. (10).

This procedure guarantees that our background estimate has a reasonably correct shape, normalization, and uncertainty. Figure 16 shows the background extrapolation for 30,300 , and $3000 \mathrm{fb}^{-1}$ together with the errors. The signal shapes are the same as in Fig. 12, and with these inputs, we 
then evaluate the expected limits for the values of integrated luminosity under consideration.

In Fig. 17, we present the 95\% CL limits for the $h_{1} h_{1} j$ and $h_{2} h_{2} j$ processes as function of $M_{h_{1}}$, together with production cross sections for $\lambda_{345}=0.019$ (red solid line) and $\lambda_{345}=1.7$ (green short dashed line) with $M_{h_{2}}=200 \mathrm{GeV}$, for both values of $\lambda_{345}$. The blue dashed line is the combined cross section for $h_{1} h_{1} j+h_{1} h_{2} j$ production for $M_{h_{2}}=M_{h_{1}}+1 \mathrm{GeV}$ and the maximal value of $\lambda_{345}^{\max }$ allowed by XENON1T data. We find that,

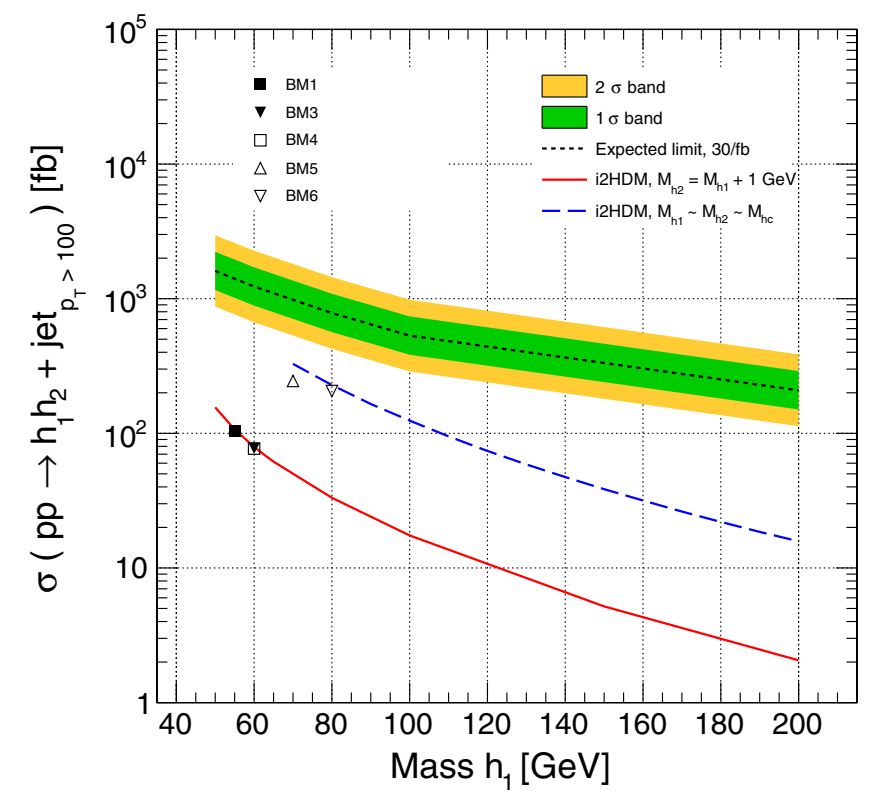

with $30 \mathrm{fb}^{-1}$ of integrated luminosity, one can exclude masses very close to $M_{\mathrm{H}} / 2$, for the maximum allowed value of $\lambda_{345}$. Further, with $3000 \mathrm{fb}^{-1}$ of the HL-LHC, one will be able to exclude all the region of $M_{h_{1}}<M_{\mathrm{H}} / 2$ for $\lambda_{345}=0.019$. At the same time, one can see that for values of $\lambda_{345}$ allowed by XENON1T LHC it will not be possible to probe $M_{h_{1}}>M_{\mathrm{H}} / 2$ with $3000 \mathrm{fb}^{-1}$ with $h_{1} h_{1} j / h_{2} h_{2} j$ process even if its cross section is maximized for $M_{h_{1}} \simeq M_{h_{2}}$. In Fig. 17, we also present the relevant benchmark points discussed in Table I. One can see that

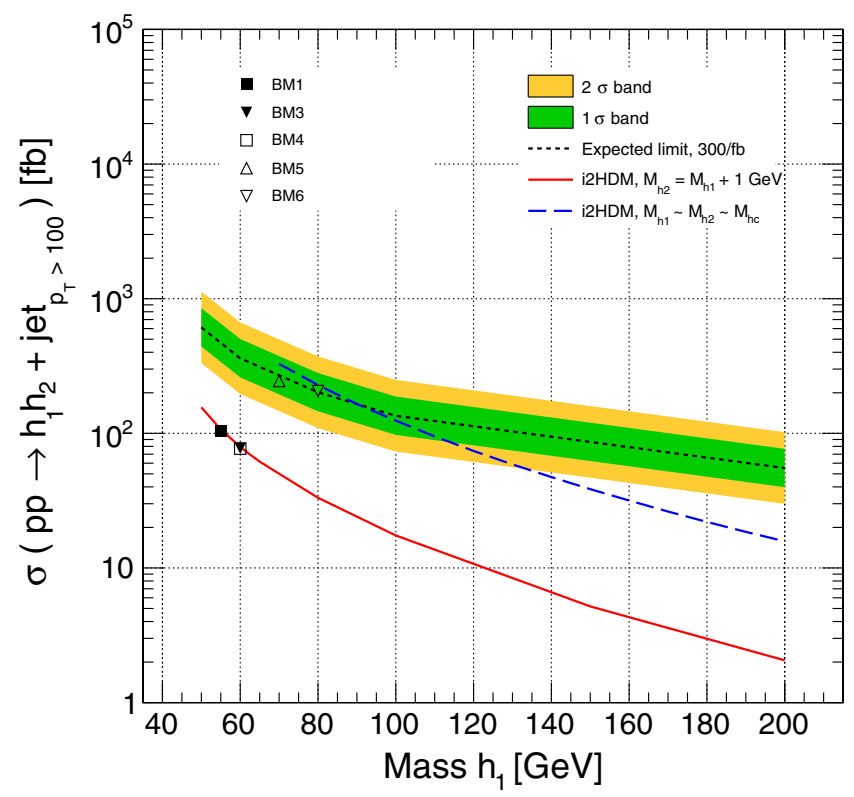

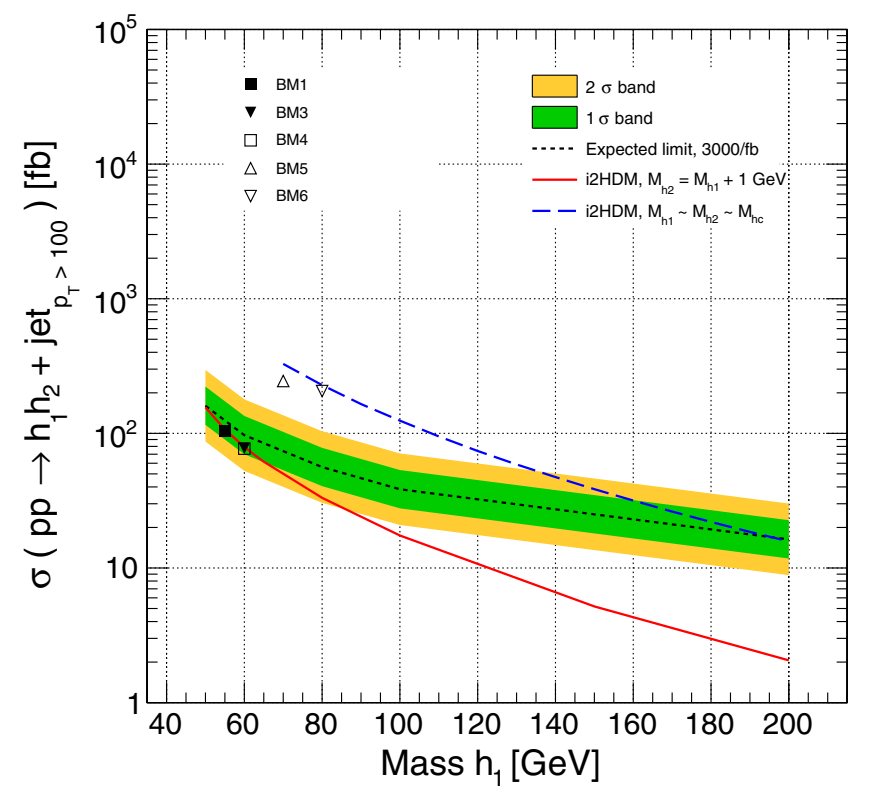

FIG. 18. Expected limits for the $h_{1} h_{2} j$ process for 30 (top left), 300 (top right), and $3000 \mathrm{fb}^{-1}$ (bottom). The red solid line is the cross section for $M_{h_{2}}=M_{h_{1}}+1 \mathrm{GeV}$. The blue short dashed line presents the cross section for the case when all inert scalars are close in mass: $M_{h_{2}}=M_{h_{c}}=M_{h_{1}}+1 \mathrm{GeV}$. The cross section is plotted for values of $M_{h_{1}}$ larger than $\sim 70 \mathrm{GeV}$, corresponding to the LEP bound for the charged Higgs [45]. The isolated symbols represent the benchmark points from Table I. All cross sections are always given for a $p_{\mathrm{T}}^{\text {jet }}>100 \mathrm{GeV}$ requirement. 
BM1 and BM3 with large (but still experimentally allowed) $\operatorname{Br}\left(H \rightarrow h_{2} h_{2}\right)$ and $\operatorname{Br}\left(H \rightarrow h_{1} h_{1}\right)$, respectively, can be probed at the LHC at high luminosity. One should note that these benchmarks predict a too-low DM relic density, requiring additional source for DM from somewhere else. At the same time, the BM2 scenario with a DM relic density, which is in agreement with the upper and lower limits from Planck Collaboration, requires, respectively, too-low values of $\lambda_{345}$ and too-low $\operatorname{Br}\left(H \rightarrow h_{1} h_{1}\right)=0.022$ to be observed at the LHC even in the high-luminosity stage. We would like to stress, however, that future DM DD experiments including XENON will be able to probe this benchmark since, as one can see from Table I, the $\sigma_{\mathrm{SI}}^{p}$ is already close to the XENON1T exclusion limit.

In Fig. 18, we present the 95\% CL limits for the $h_{1} h_{2} j$ process as function of $M_{h_{1}}$. Only for very high luminosity and for lower $M_{h_{1}} \simeq M_{h_{2}}$ masses, the LHC might be sensitive to this process alone. It is important to stress once again that this process does not depend on $\lambda_{345}$ and is therefore very complementary to the Higgs boson mediated one. One should notice that, in the $M_{h_{1}} \simeq M_{h_{2}}$ region, the actual limit should be given by a combination of this process with the $h_{1} h_{1} j$ and $h_{2} h_{2} j$ ones. The $h_{1} h_{1} j$ and $h_{2} h_{2} j$ combination is a trivial one; we just sum both cross sections, and the limit is given by Fig. 17. However, the combination with the $h_{1} h_{2} j$ process is not trivial since it has a different shape of $E_{\mathrm{T}}^{\text {miss }}$ distribution and the relative weights of $h_{1} h_{1} j / h_{2} h_{2} j$ and $h_{1} h_{2} j$ distributions are eventually dependent on the value of $\lambda_{345}$. One should also note that the sensitivity of the LHC to the $h_{1} h_{1} j / h_{2} h_{2} j$ process is very limited for $M_{h_{1}}>M_{\mathrm{H}} / 2$ as one can see from Fig. 17 since XENON1T puts a very stringent upper limit on the $\lambda_{345}$ coupling. Therefore, the $h_{1} h_{2} j$ process is likely to be a unique one for the LHC to probe the i2HDM parameter space beyond for $M_{h_{1}}>M_{\mathrm{H}} / 2$. If all (pseudo)scalar masses, $M_{h_{1}}, M_{h_{2}}$, and $M_{h^{+}}$, are similar, the LHC will be sensitive to the $M_{h_{1}}$ up to about $100 \mathrm{GeV}$ with $300 \mathrm{fb}^{-1}$ and up to about $200 \mathrm{GeV}$ with $3000 \mathrm{fb}^{-1}$ as demonstrated in the right and bottom frames of Fig. 18, respectively. The red solid line in this figure gives the cross section for $M_{h_{2}}=M_{h_{1}}+1 \mathrm{GeV}$, while the blue short dashed line is the cross section for the case when all inert scalars are close in mass $\left(M_{h_{2}}=M_{h_{c}}=M_{h_{1}}+1 \mathrm{GeV}\right)$ and the processes with the charged scalar(s) mimics the signature from the $h_{1} h_{2} j$ process. Figure 18 shows that benchmarks BM5 and BM6 with all nearly degenerate inert scalars can be tested already with $300 \mathrm{fb}^{-1}$ integrated luminosity, while BM1, $\mathrm{BM} 3$, and BM4 with nearly degenerate $h_{1}$ and $h_{2}$ can be excluded with $3000 \mathrm{fb}^{-1}$.

One can finally use the dependence of the cross section upon $\lambda_{345}$ to calculate an exclusion region on the $\left(M_{h_{1}}, \lambda_{345}\right)$ plane. Figure 19 shows the excluded values of $\lambda_{345}$ as function of $M_{h_{1}}$ for $3000 \mathrm{fb}^{-1}$. A monojet search at the HL-LHC will therefore exclude values of $\lambda_{345}$ larger than

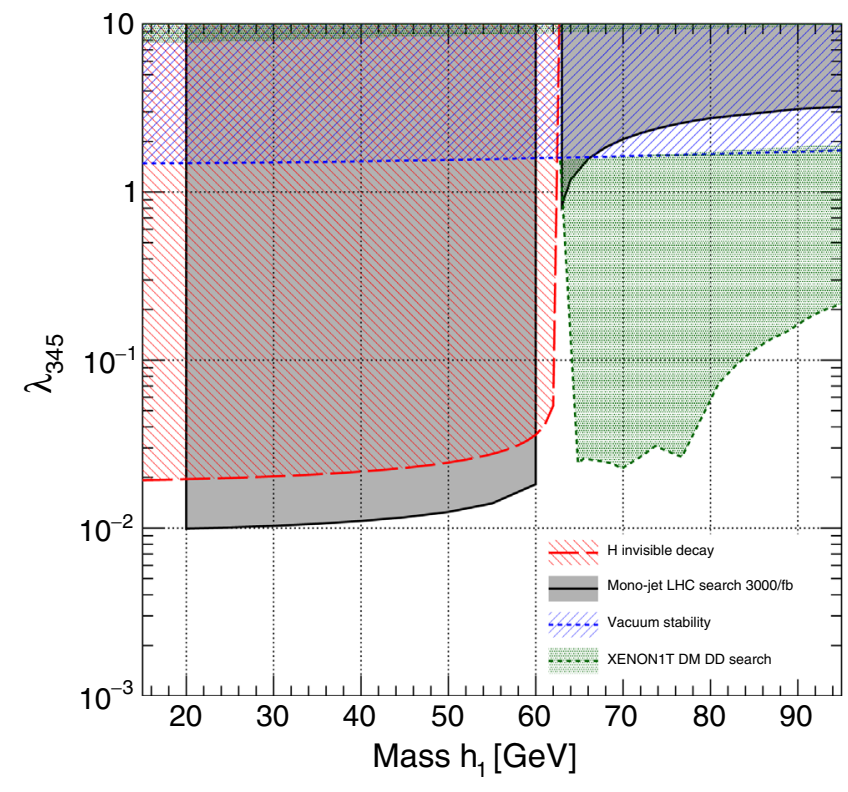

FIG. 19. Expected exclusion region on the $\left(M_{h_{1}}, \lambda_{345}\right)$ plane for $3000 \mathrm{fb}^{-1}$. The curve corresponding to Eq. (7) is given by the red dashed contor, while the expected result for $3000 \mathrm{fb}^{-1}$ is given by the black solid contour. Also shown are the limits from vacuum stability (hashed blue region, dotted contour) and the XENON1T direct detection search (shaded green region, dotted contour).

0.011-0.02, for the range of masses $M_{h_{1}}<M_{\mathrm{H}} / 2$. For higher values of $M_{h_{1}}$, one would instead need a coupling value as large as $\lambda_{345}=4.9$ in order to exclude $M_{h_{1}}<100 \mathrm{GeV}$. Also shown are the experimentally excluded regions from the invisible Higgs decay constraints as well the theoretically allowed maximum of $\lambda_{345}$ from vacuum stability.

\section{CONCLUSIONS}

In this paper, we have assessed the scope of the LHC in accessing a monojet signal stemming from the i2HDM wherein the lightest inert Higgs state $h_{1}$ is a DM candidate, produced in pairs from gluon-gluon fusion into the SM-like Higgs $H$ and accompanied by (at least) a hard jet with transverse momentum above $100 \mathrm{GeV}$, i.e., a $h_{1} h_{1} j$ final state. The second-lightest inert Higgs boson $h_{2}$ can also contribute to a monojet signature, whenever it is degenerate enough with the lightest one so that its decay products produced alongside the $h_{1}$ state are too soft to be detected. This can happen in $h_{2} h_{2} j$ (again produced by gluon-gluon fusion into the SM-like Higgs) as well as $h_{1} h_{2} j$ (induced by $\mathrm{Z}$ mediation) final states.

Before proceeding to such an assessment, we have established the viable parameter space of the i2HDM following both theoretical and experimental constraints. The former are dominated by vacuum stability requirements, whereas the latter are extracted from LEP, EWPT, LHC, relic density, as well as LUX and, especially, 
XENON1T data, which greatly reduce the accessible volume of i2HDM parameter space. The established impact of XENON1T results is in fact one of the main results of our analysis.

Over the surviving i2HDM parameter space, we have defined several benchmark points, wherein $M_{h_{1}}$ varies from 55 to $80 \mathrm{GeV}$ and $M_{h_{2}}$ is between 1 and $55 \mathrm{GeV}$ apart, and tested them against a CMS inspired selection. However, in relation to the latter, we have adopted a somewhat orthogonal approach, as we have exploited the shape of the $E_{\mathrm{T}}^{\text {miss }}$ distribution (as opposed to a standard counting experiment analysis). We have indeed shown that the shape analysis is able to obtain a better sensitivity than a counting experiment. Furthermore, we have extrapolated such sensitivity to much higher luminosities, typical of the end of the Run 2, Run 3, and high-luminosity LHC.

By adopting an improved version of standard analysis tools (i.e., matrix element, parton shower, and hadronization generators as well as detector software), which further accounts for a $k$-factor enabling us to correct the EFT approach for the emulation of the explicit loop entering the $g g \rightarrow H$ process in the signal and a sophisticated background treatment, we have been able to establish that the advocated shape analysis has significant scope in constraining monojet signals induced by i2HDM dynamics.

We have found that $h_{1} h_{1} j$ (plus $h_{2} h_{2} j$ ) and $h_{1} h_{2} j$ processes are very complementary to each other in probing the i2HDM parameter space. The former covers the $M_{h_{1}}<$ $M_{\mathrm{H}} / 2$ region and will allow us to put constraints on (in the case of void searches) or else extract (in the case of discovery) two fundamental parameters of the i2HDM entering the leading monojet process. These are the $h_{1}$ mass and the trilinear self-coupling $\lambda_{345}$ connecting the SM-like Higgs to the DM candidate pair. For example, for $M_{h_{1}}<M_{\mathrm{H}} / 2$, no values for $\lambda_{345}$ above $0.01-0.03$ would be allowed in the case of no discovery. At the same time, this process is not sensitive to $M_{h_{1}}>M_{\mathrm{H}} / 2$ for values of $\lambda_{345}$ allowed by DM DD constraints. On the other hand, $\lambda_{345^{-}}$ independent $h_{1} h_{2} j$ process can be used to probe the $M_{h_{1}}>$ $M_{\mathrm{H}} / 2$ region of the parameter space via monojet signature in case $M_{h_{1}} \simeq M_{h_{2}}$. Moreover, the $h_{1} h_{2} j$ process has a slightly less steeply falling $E_{\mathrm{T}}^{\text {miss }}$ distribution than the $h_{1} h_{1} j$ one because of different mediator $(Z$ boson instead of Higgs boson) and, consequently, a slightly better LHC limit. If all the (pseudo)scalar masses, $M_{h_{1}}, M_{h_{2}}$, and $M_{h^{+}}$, are similar, the LHC will be sensitive to $M_{h_{1}}$ up to about $100 \mathrm{GeV}$ with $300 \mathrm{fb}^{-1}$ and up to about $200 \mathrm{GeV}$ with $3000 \mathrm{fb}^{-1}$.

\section{ACKNOWLEDGMENTS}

A. B. acknowledges partial support from the STFC Grant No. ST/L000296/1, Royal Society Leverhulme Trust Senior Research Fellowship LT140094, and SotonFAPESP grant. A. B. also thanks the NExT Institute and Royal Society International Exchange Grant No. IE150682, partial support from the InvisiblesPlus RISE from the European Union Horizon 2020 research and innovation programme under the Marie SklodowskaCurie Grant No. 690575. The work of C. S. M. has been supported by the National Research Foundation of Korea (NRF) grant funded by the Korea government (MSIT) (Grants No. 2018R1A6A1A06024970 and No. 2018R1C1B5045624). S. M. is supported in part through the NExT Institute and the STFC CG ST/ L000296/1. S. M. and L. P. acknowledge funding via the H2020-MSCA-RISE-2014 Grant No. 645722 (NonMinimalHiggs). S. N., T. T., and P. M. would like to thank FAPESP for support through the Grant No. 2013/ 01907-0 and by the Cooperation Agreement (SPRINT Program) between FAPESP and the University of Southampton (U.K. Grant No. FAPESP 2013/50905-0). T. T. would additionally like to thank FAPESP for support through Grant No. 2016/15897-4.
[1] P. J. Fox, R. Harnik, J. Kopp, and Y. Tsai, Phys. Rev. D 85, 056011 (2012).

[2] A. Rajaraman, W. Shepherd, T. M. P. Tait, and A. M. Wijangco, Phys. Rev. D 84, 095013 (2011).

[3] J. Goodman, M. Ibe, A. Rajaraman, W. Shepherd, T. M. Tait, and H.-B. Yu, Phys. Rev. D 82, 116010 (2010).

[4] Y. Bai, P. J. Fox, and R. Harnik, J. High Energy Phys. 12 (2010) 048.

[5] M. Beltran, D. Hooper, E. W. Kolb, Z. A. C. Krusberg, and T. M. P. Tait, J. High Energy Phys. 09 (2010) 037.

[6] J. Goodman, M. Ibe, A. Rajaraman, W. Shepherd, T. M. P. Tait, and H.-B. Yu, Phys. Lett. B 695, 185 (2011).
[7] P. J. Fox, R. Harnik, J. Kopp, and Y. Tsai, Phys. Rev. D 84, 014028 (2011).

[8] I. M. Shoemaker and L. Vecchi, Phys. Rev. D 86, 015023 (2012).

[9] P. J. Fox and C. Williams, Phys. Rev. D 87, 054030 (2013).

[10] U. Haisch, F. Kahlhoefer, and J. Unwin, J. High Energy Phys. 07 (2013) 125.

[11] G. Busoni, A. De Simone, E. Morgante, and A. Riotto, Phys. Lett. B 728, 412 (2014).

[12] G. Busoni, A. De Simone, J. Gramling, E. Morgante, and A. Riotto, J. Cosmol. Astropart. Phys. 06 (2014) 060. 
[13] A. Belyaev, L. Panizzi, A. Pukhov, and M. Thomas, J. High Energy Phys. 04 (2017) 110.

[14] O. Buchmueller, M. J. Dolan, and C. McCabe, J High. Energy Phys. 01 (2014) 025.

[15] G. Busoni, A. De Simone, J. Gramling, E. Morgante, and A. Riotto, J. Cosmol. Astropart. Phys. 06 (2014) 060.

[16] G. Busoni, A. De Simone, T. Jacques, E. Morgante, and A. Riotto, J. Cosmol. Astropart. Phys. 09 (2014) 022.

[17] O. Buchmueller, M. J. Dolan, S. A. Malik, and C. McCabe, J High. Energy Phys. 01 (2015) 037.

[18] M. R. Buckley, D. Feld, and D. Goncalves, Phys. Rev. D 91, 015017 (2015).

[19] J. Abdallah et al., Phys. Dark Universe 9-10, 8 (2015).

[20] J. Abdallah, A. Ashkenazi, A. Boveia, G. Busoni, A. De Simone et al., arXiv:1409.2893.

[21] D. Abercrombie et al., arXiv:1507.00966.

[22] N. G. Deshpande and E. Ma, Phys. Rev. D 18, 2574 (1978).

[23] E. Ma, Phys. Rev. D 73, 077301 (2006).

[24] R. Barbieri, L. J. Hall, and V. S. Rychkov, Phys. Rev. D 74, 015007 (2006).

[25] L. Lopez Honorez, E. Nezri, J. F. Oliver, and M. H. Tytgat, J. Cosmol. Astropart. Phys. 02 (2007) 028.

[26] C. Arina, F.-S. Ling, and M. H. G. Tytgat, J. Cosmol. Astropart. Phys. 10 (2009) 018.

[27] E. Nezri, M. H. G. Tytgat, and G. Vertongen, J. Cosmol. Astropart. Phys. 04 (2009) 014.

[28] X. Miao, S. Su, and B. Thomas, Phys. Rev. D 82, 035009 (2010).

[29] M. Gustafsson, S. Rydbeck, L. Lopez-Honorez, and E. Lundstrom, Phys. Rev. D 86, 075019 (2012).

[30] A. Arhrib, R. Benbrik, and N. Gaur, Phys. Rev. D 85, 095021 (2012).

[31] B. Swiezewska and M. Krawczyk, Phys. Rev. D 88, 035019 (2013).

[32] A. Goudelis, B. Herrmann, and O. Stål, J. High Energy Phys. 09 (2013) 106.

[33] A. Arhrib, Y.-L. S. Tsai, Q. Yuan, and T.-C. Yuan, J. Cosmol. Astropart. Phys. 06 (2014) 030.

[34] M. Krawczyk, D. Sokolowska, P. Swaczyna, and B. Swiezewska, J. High Energy Phys. 09 (2013) 055.

[35] M. Krawczyk, D. Sokołowska, P. Swaczyna, and B. Świeżewska, Acta Phys. Pol. B 44, 2163 (2013).

[36] A. Ilnicka, M. Krawczyk, and T. Robens, Phys. Rev. D 93, 055026 (2016).

[37] M. A. Díaz, B. Koch, and S. Urrutia-Quiroga, Adv. High Energy Phys. 2016, 8278375 (2016).

[38] K. P. Modak and D. Majumdar, Astrophys. J. Suppl. Ser. 219, 37 (2015).

[39] F. S. Queiroz and C. E. Yaguna, J. Cosmol. Astropart. Phys. 02 (2016) 038.

[40] C. Garcia-Cely, M. Gustafsson, and A. Ibarra, J. Cosmol. Astropart. Phys. 02 (2016) 043.

[41] M. Hashemi and S. Najjari, Eur. Phys. J. C 77, 592 (2017).

[42] P. Poulose, S. Sahoo, and K. Sridhar, Phys. Lett. B 765, 300 (2017).

[43] A. Alves, D. A. Camargo, A. G. Dias, R. Longas, C. C. Nishi, and F. S. Queiroz, J. High Energy Phys. 10 (2016) 015.

[44] A. Datta, N. Ganguly, N. Khan, and S. Rakshit, Phys. Rev. D 95, 015017 (2017).
[45] A. Belyaev, G. Cacciapaglia, I. P. Ivanov, F. Rojas-Abatte, and M. Thomas, Phys. Rev. D 97, 035011 (2018).

[46] G. Aad et al. (ATLAS Collaboration), J. High Energy Phys. 01 (2016) 172.

[47] V. Khachatryan et al. (CMS Collaboration), J. High Energy Phys. 02 (2017) 135.

[48] A. Belyaev, N. D. Christensen, and A. Pukhov, Comput. Phys. Commun. 184, 1729 (2013).

[49] G. Belanger, F. Boudjema, A. Pukhov, and A. Semenov, Comput. Phys. Commun. 149, 103 (2002).

[50] G. Belanger, F. Boudjema, A. Pukhov, and A. Semenov, Comput. Phys. Commun. 174, 577 (2006).

[51] P. A. R. Ade et al. (Planck Collaboration), Astron. Astrophys. 571, A16 (2014).

[52] P. A. R. Ade et al. (Planck Collaboration), Astron. Astrophys. 594, A13 (2016).

[53] D. S. Akerib et al. (LUX Collaboration), Phys. Rev. Lett. 112, 091303 (2014).

[54] E. Aprile et al. (XENON Collaboration), Phys. Rev. Lett. 119, 181301 (2017).

[55] Digitized XENON1T results at PhenoData project, https:// hepmdb.soton.ac.uk/phenodata/view.php? $\mathrm{id}=595 \mathrm{e} 239 \mathrm{abb} 817586383 \mathrm{e} 929 \mathrm{~d}$.

[56] A. Ilnicka, T. Robens, and T. Stefaniak, Mod. Phys. Lett. A 33, 1830007 (2018).

[57] M. Bondarenko, A. Belyaev, J. Blandford, L. Basso, E. Boos, V. Bunichev et al., arXiv:1203.1488.

[58] R. D. Ball et al., Nucl. Phys. B867, 244 (2013).

[59] D. de Florian et al. (LHC Higgs Cross Section Working Group), arXiv:1610.07922.

[60] J. Alwall, M. Herquet, F. Maltoni, O. Mattelaer, and T. Stelzer, J. High Energy Phys. 06 (2011) 128.

[61] J. Alwall, R. Frederix, S. Frixione, V. Hirschi, F. Maltoni, O. Mattelaer, H. S. Shao, T. Stelzer, P. Torrielli, and M. Zaro, J. High Energy Phys. 07 (2014) 079.

[62] J. M. Lindert, K. Kudashkin, K. Melnikov, and C. Wever, Phys. Lett. B 782, 210 (2018).

[63] S. P. Jones, M. Kerner, and G. Luisoni, Phys. Rev. Lett. 120, 162001 (2018).

[64] T. Neumann, J. Phys. Commun. 2, 095017 (2018).

[65] T. Sjostrand, S. Mrenna, and P. Z. Skands, J. High Energy Phys. 05 (2006) 026.

[66] T. Sjostrand, S. Mrenna, and P.Z. Skands, Comput. Phys. Commun. 178, 852 (2008).

[67] A. Buckley, J. Ferrando, S. Lloyd, K. Nordström, B. Page, M. Rüfenacht, M. Schönherr, and G. Watt, Eur. Phys. J. C 75, 132 (2015).

[68] J. de Favereau, C. Delaere, P. Demin, A. Giammanco, V. Lemaître, A. Mertens, and M. Selvaggi (DELPHES 3 Collaboration), J. High Energy Phys. 02 (2014) 057.

[69] A. L. Read, J. Phys. G 28, 2693 (2002).

[70] G. Cowan, K. Cranmer, E. Gross, and O. Vitells, Eur. Phys. J. C 71, 1554 (2011); 73, 2501(E) (2013).

[71] T. Müller, J. Ott, and J. Wagner-Kuhr, http://www-ekp .physik.uni-karlsruhe.de/ ott/theta/theta-auto/index.html.

[72] (CMS Collaboration) Report No. CMS-PAS-EXO-16-013, 2016.

[73] V. Khachatryan et al. (CMS Collaboration), Eur. Phys. J. C 75, 235 (2015). 